\title{
QoS Provisioning in a Scalable Wireless Mesh Network for Intelligent Transportation Systems
}

\author{
Jane-Hwa Huang, Li-Chun Wang, Senior Member, IEEE, and Chung-Ju Chang, Fellow, IEEE
}

\begin{abstract}
The wireless mesh network (WMN) is an economical low-power solution to provide wireless broadband access in intelligent transportation systems (ITSs). This paper investigates how to deploy access points (APs) to improve throughput and qualityof-service $(\mathrm{QoS})$, when the multihop communication is used to extend the coverage of wireless ITS network. Frequency planning is suggested to improve the capacity with QoS provisioning and to make the system more scalable. To investigate the overall tradeoffs among QoS, throughput, and coverage, we develop an analytical model to evaluate throughput, frame delay, and jitter for the considered ITS WMN using the carrier-sense multiple-access (CSMA) medium access control (MAC) protocol. Then, we apply an optimization approach to determine the optimal separation distances between APs with the delay requirement. To provide a guideline for network planning, we compare the uniform-spacing and increasing-spacing AP deployment strategies. The uniformspacing strategy is to make all the APs with the same separation distance. In the increasing-spacing strategy, the separation distances between APs are increased from the central AP to the outer APs. Because of the shorter separation distance, the AP closer to the central AP can deliver higher traffic. It is shown that the increasing-spacing strategy outperforms the uniform-spacing strategy in terms and a profit function, considering the capacity and the total cost for deploying APs.
\end{abstract}

Index Terms-Intelligent transportation system (ITS), qualityof-service (QoS), wireless mesh network (WMN).

\section{INTRODUCTION}

D UE to the advantages of easy deployment and lower cost, the wireless mesh network (WMN) is a promising information dissemination technology for intelligent transportation systems (ITSs) [1]-[5]. The ITS development aims to integrate communication, information, and control technologies to improve the safety and convenience of transportation systems. In wireless-enabled ITS systems, the users can conveniently retrieve public safety warning, traveller and traffic information, and rich media content [6], [7]. Therefore, widely deploying ITS wireless networks has become a critical topic. Since a

Manuscript received February 23, 2007; revised July 4, 2007, November 4, 2007, and December 31, 2007. This work was supported in part by the MoE ATU Plan, by the Program for Promoting Academic Excellence of Universities (Phase II), and by the National Science Council under Grant 95W803C, Grant NSC 95-2752-E-009-014-PAE, Grant NSC 95-2221-E-009148, and Grant NSC 95-2221-E-009-155. This work was presented in part at the IEEE Conference on Systems, Man, and Cybernetics (SMC) 2006, Taipei, Taiwan, R.O.C., October 2006. The review of this paper was coordinated by Prof. X. Shen

The authors are with the Department of Communication Engineering, National Chiao Tung University, Hsinchu 300, Taiwan, R.O.C. (e-mail: hjh@mail.nctu.edu.tw; lichun@cc.nctu.edu.tw; cjchang@cc.nctu.edu.tw).

Color versions of one or more of the figures in this paper are available online at http://ieeexplore.ieee.org.

Digital Object Identifier 10.1109/TVT.2008.918701 multihop architecture can avoid costly and difficult cabling engineering, the WMN is an economical low-power solution to support ubiquitous ITS broadband applications.

Fig. 1 illustrates a cluster-based ITS WMN for the denseurban scenario. To provide reliable broadband communications, the access points (APs) are deployed along the main streets as in [8]. The APs are connected via wireless links to facilitate deployment. Moreover, the APs are grouped into clusters. In each cluster, only the central AP has a wireline connection to the Internet, whereas other APs will relay the neighboring APs' traffic toward/from the central AP. By this multihop architecture, ITS WMNs can rapidly be deployed on a large scale with less cabling engineering.

Nevertheless, WMNs face scalability issues since coverage extension, throughput enhancement, and delay improvement are usually contradictory goals [1]-[3], [9], [10]. Specifically, multihop communications can extend coverage by more hops and longer hop distance. Maximizing the coverage of AP can reduce infrastructure cost. However, the repeatedly relayed traffic with more hops may rapidly exhaust radio resources and degrade quality-of-service (QoS), e.g., longer delay. The longer hop distance will also lower the data rate in the relay link between APs. Meanwhile, the increasing collisions due to more users in a larger coverage of AP will further lower user throughput. Therefore, while multihop communication is used to extend coverage, how to improve throughput and QoS is a major concern for deploying ITS WMNs.

In the literature, the AP deployment issues for wireless local area networks (WLANs) are studied in [11]-[14]. In [11], an optimization approach is applied to minimize the area with poor signal quality. The authors in [12] and [13] have developed optimization algorithms to minimize the bit error rate. In [14], an optimal AP deployment problem is formulated, which aims to minimize the maximum of channel utilization to achieve load balancing. In [11]-[14], all the APs are connected via cables. The performance of WMNs is investigated in [1], [3], [9], [10], and [15]-[17]. The simulation results in [9] and the experimental results in [10] show that even if only one user is sending data, the user throughput rapidly drops as the number of hops increases from one. Then, it stabilizes at a very low throughput as the number of hops becomes larger (e.g., larger than seven [9]). This phenomenon is due to the fact that adjacent hop nodes have to contend for the channel to relay traffic. The authors in [15] and [16] point out that with $k$ users in an ad hoc network, the user throughput is scaled like $O(1 / \sqrt{k \log k})$. It is also shown in [3] that the user throughput in a WMN sharply decreases as $O(1 / k)$ due to the throughput bottleneck at the central AP. A few papers have considered the overall 


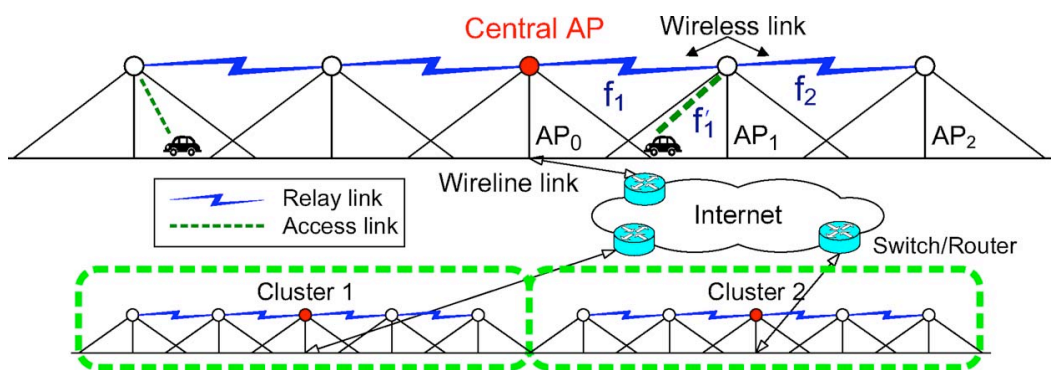

Fig. 1. System architecture for ITS WMN. (This is an example for the uniform-spacing deployment strategy.)
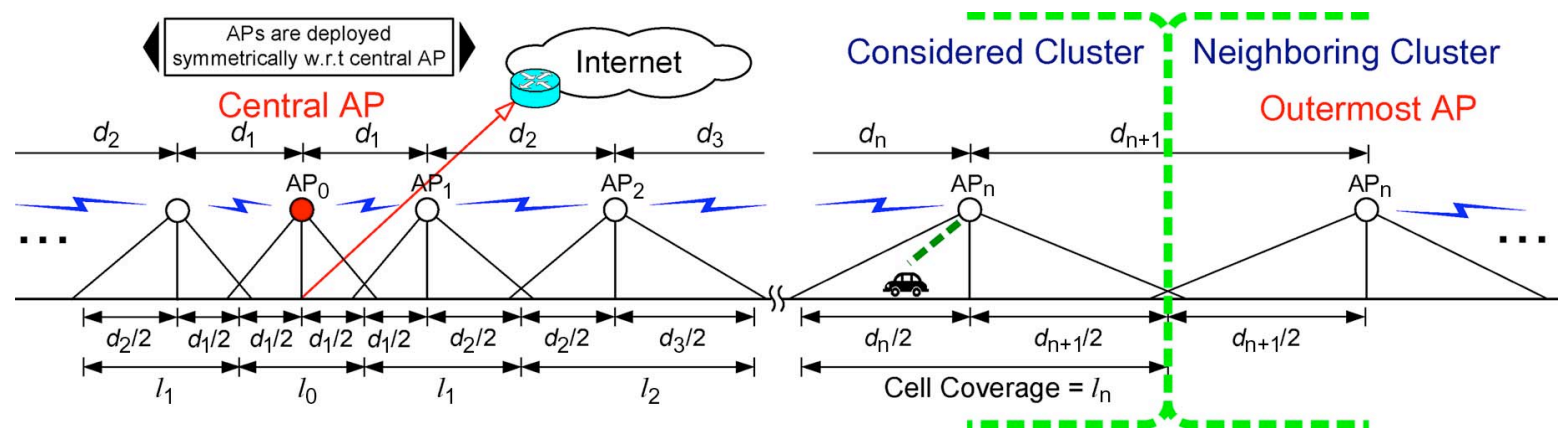

Fig. 2. Cluster of APs in ITS WMN, where only the single side of a cluster is shown since the APs in the other side are symmetrically deployed. (This is an example for the increasing-spacing deployment strategy.)

throughput and coverage performance issues when deploying APs in ITS WMNs. The work in [1] studies the throughput and coverage of WMN in a single-user case. In [17], the tradeoff between throughput and coverage for a multiuser ITS WMN is investigated. However, the QoS (delay and jitter) issues are not considered in [1] and [17].

This paper investigates the AP deployment issue in a scalable ITS WMN, as shown in Fig. 1. We suggest allocating each AP with different channels. This simple frequency planning can reduce contention collisions to improve throughput and make the WMN more scalable. The cluster-based structure also facilitates the management of QoS and throughput in a WMN since we can adjust the separation distances between APs to control the data rate of relay link and the number of users served by an AP. To investigate the overall tradeoffs among throughput, coverage, and delay, we develop an analytical model to evaluate the throughput and delay for the considered multihop network employing the carrier-sense multiple-access (CSMA) medium access control (MAC) protocol. The developed model considers a practical case where all nodes have bidirectional asymmetric traffic load and operate in the unsaturated situation. That is, the node does not always have a frame waiting for transmission. On top of the analytical model, we apply an optimization approach to determine the best number of APs in a cluster and the optimal separation distances between APs. The objective aims to maximize the profit function defined as the ratio of the capacity to the total cost for deploying a cluster of APs with the QoS requirement. To provide a useful guideline for network planning, we compare the uniform-spacing and increasingspacing AP deployment strategies. In the uniform-spacing strategy (see Fig. 1), all APs have the same separation distance. In the increasing-spacing strategy (in Fig. 2), the separation distances between APs are increased from the central AP to the outer APs. We respectively determine the optimal deployment parameters for these strategies and compare their performances.

The analytical model is validated by simulations. It is shown that although there are some assumptions and approximations, the analytical model nicely estimates the link capacity. Moreover, the analytical model can estimate the upper bound of traffic load subject to delay requirement. With the help of this analytical model, we can analytically determine the optimal deployment parameters to maximize the profit of a cluster with the QoS requirement.

The rest of this paper is organized as follows. Section II describes the system architecture and the traffic model for an ITS WMN. In Section III, we formulate an optimization problem to determine the optimal deployment parameters with the delay constraint. Sections IV and V describe the analytical throughput and delay model for the considered ITS WMN. Performance evaluations are shown in Section VI. Concluding remarks are given in Section VII.

\section{SCALABLE ITS WMN}

\section{A. Network Architecture and Assumptions}

Fig. 1 shows a scalable cluster-based ITS WMN for denseurban coverage. The APs are deployed along the main streets to avoid heavy attenuation due to buildings and walls. In a cluster, the $i$ th $\mathrm{AP}\left(A P_{i}\right)$ from the central AP $\left(A P_{0}\right)$ connects to $A P_{0}$ via an $i$-hop communication, and only the central AP is Internet connected through cables. Clearly, this WMN is very suitable for wireless-enabled ITS systems thanks to easy deployment and less cost.

This ITS WMN operates in a multichannel and multiinterface fashion. The APs are allocated with different channels to reduce the number of contending users. Moreover, each AP has three 


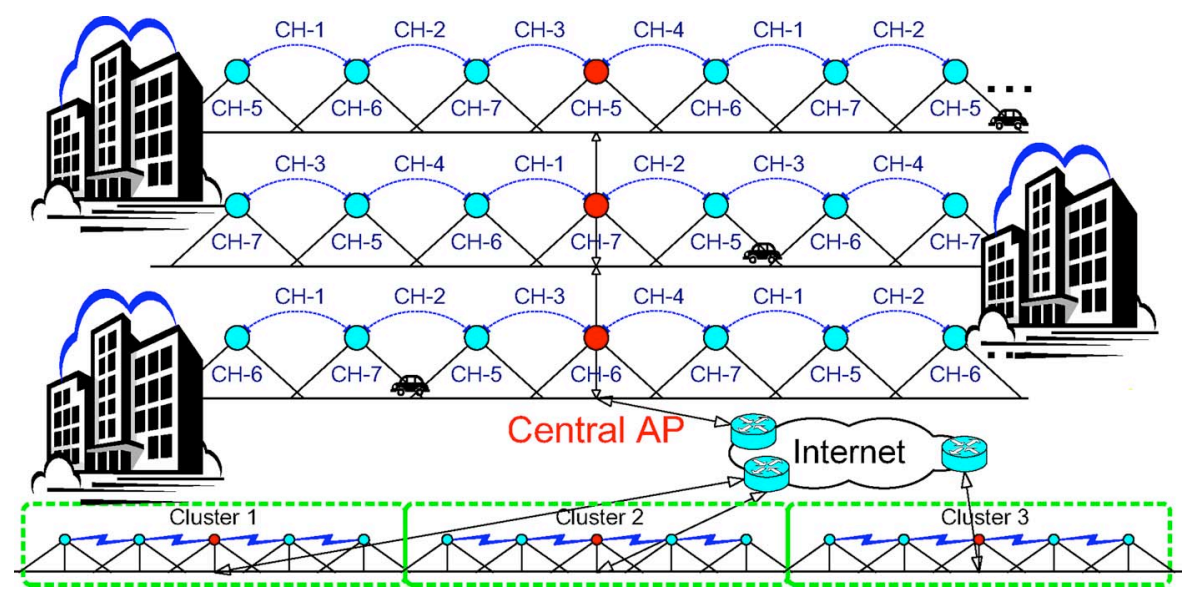

Fig. 3. Example of frequency assignment in ITS WMN, where seven nonoverlapping channels ensure two buffer cells.

radio interfaces: one for data access and two for data forwarding. By doing so, an AP can concurrently provide data access for local users at channel $f_{i}^{\prime}$ and receive/deliver relay traffic from/to $A P_{i-1}$ and $A P_{i+1}$ at different channels $f_{i}$ and $f_{i+1}$, respectively, thereby reducing delay.

In general, spectrum and hardware costs are the major concerns in the multichannel and multiinterface systems. However, there are multiple channels available for wireless public access. For example, there are 12 nonoverlapping channels for IEEE 802.11a WLAN, three channels for IEEE 802.11b/g WLAN, and $75 \mathrm{MHz}$ of spectrum reserved for the dedicated short-range communication (DSRC) $)^{1}$ [6], [7]. The price of interface also rapidly goes down since the WLAN has become an off-theshelf product. Indeed, many WLAN equipment vendors have developed the outdoor IEEE $802.11 \mathrm{a} / \mathrm{b} / \mathrm{g}$ multimode APs with multiple interfaces [18].

Frequency planning in this ITS WMN is simple since it only needs to design the separation distances between APs to ensure a sufficient cochannel reuse distance without interference. Fig. 3 shows an example of frequency assignment. Let the term cell represent the coverage of an AP. In Fig. 3, seven channels can ensure two buffer cells between two cochannel cells. We also assume that the interference from adjacent streets is very minor since it is heavily attenuated by buildings. In practice, other WLAN users may interfere with this ITS WMN operating at the unlicensed band. In this situation, we suggest allocating the channels in the licensed DSRC band for the relay links between APs to ensure throughput. To understand performance bounds, this interference issue is not considered in this paper.

\section{B. Uniform-Spacing and Increasing-Spacing AP Deployment Strategies}

To provide a useful insight into AP deployment, we compare the uniform-spacing and increasing-spacing strategies. The uniform-spacing strategy is to make all the APs have the same separation distance as shown in Fig. 1. According to the increasing-spacing strategy as in Fig. 2, the separation

${ }^{1}$ DSRC is a short-to-medium-range communication system for ITS wireless applications, which supports both public safety and private operations in vehicle-to-roadside and vehicle-to-vehicle environments [7]. distances $d_{i}$ between APs follow the order $d_{1} \leq d_{2} \leq \cdots \leq d_{n}$. In a WMN, the AP closer to the central AP aggregates more traffic. With a shorter separation distance, the AP near the central AP can deliver higher traffic load for a cluster.

\section{Traffic Model for User and AP in an ITS WMN}

This paper considers bidirectional asymmetric traffic. The demanded uplink and downlink traffic of a user are $r_{U}$ and $r_{D}$ (in megabits per second), respectively. Suppose that all the APs have the same transmission power. We also assume an ideal handoff mechanism, and the user always associates with the closer AP by estimating the average signal strength from APs. As in Fig. 2, the separation distance between $A P_{i-1}$ and $A P_{i}$ is $d_{i}$, and the distance from $A P_{n}$ to the outermost $\mathrm{AP}$ in the neighboring cluster is $d_{n+1}$. Then, the coverage of $A P_{i}$ is defined as $l_{i}=\left(d_{i}+d_{i+1}\right) / 2$, and clearly, $l_{0}=d_{1}$. Let $D_{M}$ (users $/ \mathrm{m}$ ) be the user density. The aggregated uplink and downlink access traffic of $A P_{i}$ are $R_{U, i}^{(A)}=l_{i} D_{M} r_{U}$ and $R_{D, i}^{(A)}=l_{i} D_{M} r_{D}$, respectively.

Fig. 4 depicts the traffic model for the AP and users in an ITS WMN. Let $L$ be the frame payload size. The frame arrival rate and the service rate of a user are $\lambda_{k}=r_{U} / L$ and $\mu_{k}$ (in frames per second), respectively. The traffic load of an AP includes the access traffic for local users and the relay traffic for other APs. To facilitate management, each AP has three queues dedicated for downlink access traffic, uplink relay traffic, and downlink relay traffic, respectively. The aggregated downlink access frame arrival rate of $A P_{i}$ is $\lambda_{D, i}^{(A)}=R_{D, i}^{(A)} / L=$ $l_{i} D_{M} r_{D} / L$. The uplink and downlink relay frame arrival rates of $A P_{i}$ are equal to

$$
\begin{aligned}
& \lambda_{U, i}^{(R)}=\frac{R_{U, i}^{(R)}}{L}=\frac{\left(R_{U, i}^{(A)}+R_{U, i+1}^{(R)}\right)}{L}=\frac{\sum_{i^{\prime}=i}^{n} l_{i^{\prime}} D_{M} r_{U}}{L} \\
& \lambda_{D, i}^{(R)}=\frac{R_{D, i}^{(R)}}{L}=\frac{\sum_{i^{\prime}=i+1}^{n} R_{D, i^{\prime}}^{(A)}}{L}=\frac{\sum_{i^{\prime}=i+1}^{n} l_{i^{\prime}} D_{M} r_{D}}{L}
\end{aligned}
$$

where $n$ is the number of APs in the single side of a cluster; $R_{U, i}^{(R)}=\sum_{i^{\prime}=i}^{n} R_{U, i^{\prime}}^{(A)}$ is the aggregated uplink relay traffic from the users served by $A P_{i}, A P_{i+1}, \ldots, A P_{n}$; and $R_{D, i}^{(R)}$ is the aggregated downlink relay traffic. The frame service rates are 


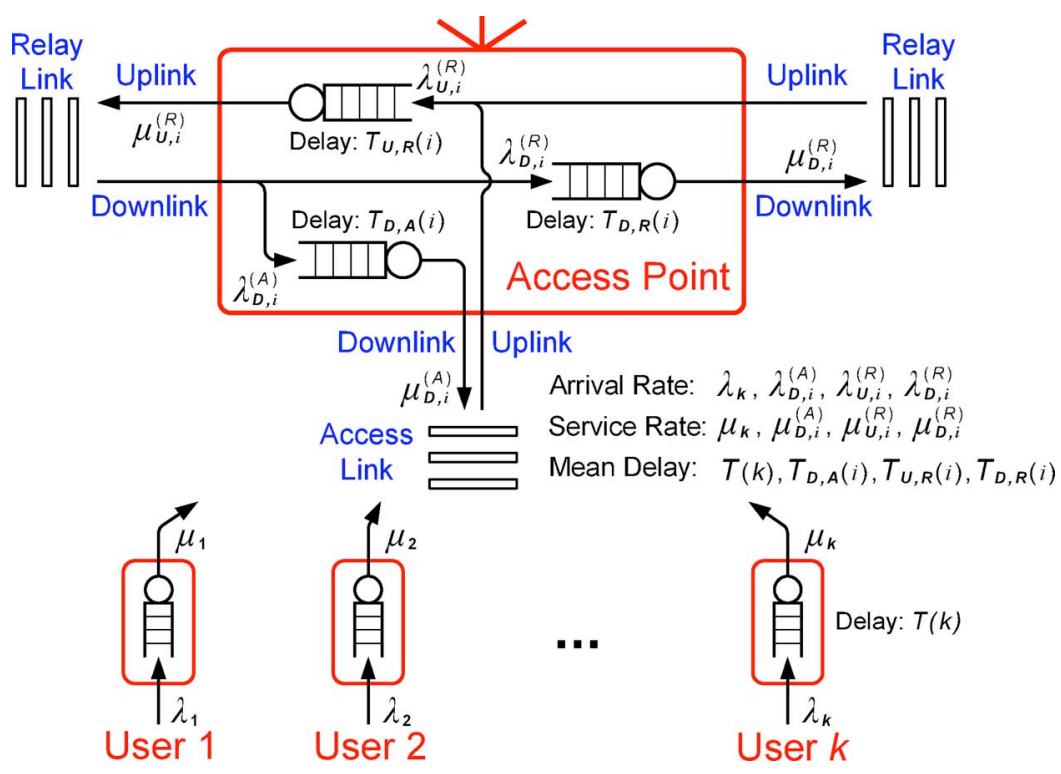

Fig. 4. Traffic models for users and the AP. For management simplicity, each AP has three isolated queues for downlink access traffic, uplink relay traffic, and downlink relay traffic, respectively.

TABLE I

LIST OF NOTATIONS

\begin{tabular}{cl}
\hline Symbol & Item (DL: downlink, UL: uplink) \\
\hline$i, i^{\prime}$ & The index of AP \\
$j, j^{\prime}, k$ & The index of node \\
$n$ & The number of APs in a cluster \\
$K$ & Total number of contending nodes \\
$d_{i}$ & Separation distance between $A P_{i-1}$ and $A P_{i}(\mathrm{~m})$ \\
$l_{i}$ & Coverage of $A P_{i}(\mathrm{~m})$ \\
$D_{M}$ & User density (users/m) \\
$L$ & Data frame payload size (Bytes) \\
$r_{D}, r_{U}$ & Demanded DL/UL traffic of each user $(\mathrm{Mb} / \mathrm{s})$ \\
$R_{D, i}^{(A)}, R_{U, i}^{(A)}$ & Aggregated DL/UL access traffic of $A P_{i}(\mathrm{Mb} / \mathrm{s})$ \\
$R_{D, i}^{(R)}, R_{U, i}^{(R)}$ & Aggregated DL/UL relay traffic of $A P_{i}(\mathrm{Mb} / \mathrm{s})$ \\
$\lambda_{k}, \mu_{k}$ & UL access frame arrival/service rate of node $k(f r a m e s / \mathrm{s})$ \\
$\lambda_{D, i}^{(A)}, \mu_{D, i}^{(A)}$ & DL access frame arrival/service rate of $A P_{i}($ frames $/ \mathrm{s})$ \\
$\lambda_{U, i}^{(R)}, \mu_{U, i}^{(R)}$ & UL relay frame arrival/service rate of $A P_{i}($ frames $/ \mathrm{s})$ \\
$\lambda_{D, i}^{(R)}, \mu_{D, i}^{(R)}$ & DL relay frame arrival/service rate of $A P_{i}($ frames $/ \mathrm{s})$ \\
\hline$T_{t}$ & Duration of channel activity type $t(\mathrm{~s})$ \\
$T_{S}, T_{C}$ & Successful frame transmission time/Collision duration $(\mathrm{s})$ \\
$T_{v, k}$ & Average duration of activity slot observed by node $k(\mathrm{~s})$ \\
$\nu_{t, k}$ & Channel-activity-type probability observed by node $k$ \\
$\rho_{k}$ & Busy probability of node $k$ \\
$\tau_{k}$ & Conditional transmission probability of node $k$ \\
$p_{k}$ & Unsuccessful frame transmission probability of node $k$ \\
\hline &
\end{tabular}

$\mu_{D, i}^{(A)}, \mu_{U, i}^{(R)}$, and $\mu_{D, i}^{(R)}$, respectively. For clarity, the notations in the traffic model are summarized in Table I.

\section{Scalability and $Q o S$}

Most traditional WMNs are not scalable. As the number of users increases, user throughput and QoS (delay) sharply degrade due to the increasing collisions [3], [9], [10], [15], [16]. By contrast, the presented ITS WMN is more scalable to accommodate more users because frequency planning can reduce the number of contending nodes to improve contention situation. Furthermore, delay and throughput can be guaranteed by properly designing the number of APs in a cluster and the separation distances of APs. The remaining challenge lies in the way to determine the optimal separation distances between APs to achieve the optimal tradeoff among QoS, throughput, and coverage.

\section{A. Problem Formulation}

All the performance issues of coverage, throughput, and QoS are essential factors in designing a scalable ITS WMN. From the coverage viewpoint, a larger separation distance between APs can lower the infrastructure cost due to fewer APs. From the throughput standpoint, however, a shorter separation distance can achieve a higher relay link capacity between APs. Meanwhile, a smaller cell with fewer contending users also improves the access link capacity between users and the AP. We also consider the frame delay consisting of contention delay and queuing delay. From the queuing delay perspective, a longer separation distance of AP is better due to fewer hops. From the contention delay viewpoint, a shorter separation distance and then a smaller cell may be preferred due to higher data rate and fewer contending users. In the following, an optimization problem is formulated to determine the best number of APs in a cluster and the optimal separation distances between APs subject to the constraints on throughput and delay.

First, we discuss the constraints in the optimization problem for the considered ITS WMN.

1) The frame arrival rate for each user and AP should be less than the frame service rate to guarantee minimum throughput. Therefore, for each user, it is required that

$$
\lambda_{k} \leq \mu_{k}
$$

and for each AP

$\lambda_{D, i}^{(A)} \leq \mu_{D, i}^{(A)}, \quad \lambda_{U, i}^{(R)} \leq \mu_{U, i}^{(R)}, \quad \lambda_{D, i}^{(R)} \leq \mu_{D, i}^{(R)}$.

These constraints also mean that the link capacity of each node is large enough to accommodate its traffic load. 
2) The overall frame delay $D_{T}(i)$ for the user in the cell of $A P_{i}$ should meet the delay requirement $D_{\text {req }}$. Accordingly

$$
D_{T}(i) \leq D_{\text {req }}
$$

3) The cell coverage of an AP should be designed to ensure an acceptable data rate in the access link. Recall that the coverage of $A P_{i}$ is $l_{i}=\left(d_{i} / 2+d_{i+1} / 2\right)$. The maximum possible separation distance between a user and the AP should be less than a threshold $r_{\text {MAX }}$. That is

$$
\max \left(d_{i} / 2, d_{i+1} / 2\right) \leq r_{\text {MAX }}
$$

4) The separation distance between APs should be designed from two folds. The separation distance $d_{i}$ should be less than the maximal reception range $d_{\mathrm{MAX}}$ while larger than $d_{\mathrm{MIN}}$ to lower the handoff probability and ensure sufficient cochannel reuse distance without interference. Hence,

$$
\left\{\begin{array}{l}
d_{\mathrm{MIN}} \leq d_{i} \leq d_{\mathrm{MAX}}, \quad \text { for } i=1,2, \ldots, n \\
d_{\mathrm{MIN}} \leq d_{n+1}
\end{array}\right.
$$

where $d_{n+1}$ is the separation distance from $A P_{n}$ to the outermost AP in the neighboring cluster, as in Fig. 2.

According to the above considerations, the AP deployment issue can be formulated as a mixed-integer nonlinear programming problem with the following decision variables: $n$ (the number of APs in the single side of one cluster) and $d_{i}$ (the separation distances between APs). In this scalable WMN, frequency planning reduces the number of contending users to relieve the collision issue as the coverage of a cluster increases. The optimal coverage and capacity can simultaneously be achieved since more users in a cluster can also lead to higher capacity of a cluster. It is noteworthy that due to the limitation of relay link capacity between APs, deploying more APs in a cluster may not significantly improve the capacity of a cluster. Therefore, the optimization problem aims to maximize the profit of a cluster defined as the ratio of the capacity to the cost for deploying a cluster of APs.

The capacity of a cluster is defined as the total aggregated traffic of the central AP $\left(A P_{0}\right)$, including the relay traffic from/to other APs and the local access traffic for the users served by $A P_{0}$. According to the constraints in (3) and (4), the link capacity of each node is large enough to accommodate the carried traffic load. Hence, the aggregated traffic of $A P_{0}$ is equal to the sum of demanded traffic of all users. Consider that a cluster includes $A P_{0}$ and $2 n$ APs deployed symmetrically with respect to $A P_{0}$. The coverage of a cluster is $\left(2 \sum_{i=1}^{n} d_{i}+\right.$ $\left.d_{n+1}\right)$. Let $D_{M}$ (users/m) be the user density, and let $r_{U}$ and $r_{D}$ be the demanded uplink and downlink traffic of each user, respectively. Then, the capacity of a cluster is

$$
\left(2 \sum_{i=1}^{n} d_{i}+d_{n+1}\right) D_{M}\left(r_{U}+r_{D}\right) .
$$

The deployment cost means the investment for deploying a cluster of APs, including the expense of devices and that of cabling engineering work. The more APs in a cluster, the higher the deployment cost of a cluster. Suppose that $c_{\mathrm{AP}}$ is the cost of an AP and that $\rho_{l}$ is the wireline overhead for connecting $A P_{0}$ to the Internet. Since a cluster consists of $(2 n+1)$ APs, the deployment cost of a cluster is $(2 n+1) c_{\mathrm{AP}}+\rho_{l}$. For convenience, the cost of a cluster is normalized to the cost of an AP. Then, the normalized deployment cost of a cluster can be expressed as $(2 n+1+\rho)$, where $\rho=\rho_{l} / c_{\mathrm{AP}}$.

In the following, we discuss the optimal AP deployment problems for the increasing-spacing and uniform-spacing strategies.

\section{B. Increasing-Spacing Deployment Strategy}

In the increasing-spacing strategy, the separation distances of APs follow the order $d_{1} \leq d_{2} \leq \cdots \leq d_{n}$. That is, the heavier the load of AP, the shorter the separation distance. The optimal deployment parameters can be determined by solving the following optimization problem:

$$
\begin{gathered}
\underset{d_{1}, d_{2}, \ldots, d_{n+1}}{\operatorname{MAX}} \frac{\text { Capacity of a cluster of APs }}{\text { Total cost for deploying a cluster of APs }} \\
=\underset{n, d_{1}, d_{2}, \ldots, d_{n+1}}{\operatorname{MAX}} \frac{\left(2 \sum_{i=1}^{n} d_{i}+d_{n+1}\right) D_{M}\left(r_{U}+r_{D}\right)}{(2 n+1+\rho)}
\end{gathered}
$$

subject to

$$
\begin{aligned}
& \lambda_{k} \leq \mu_{k}, \lambda_{D, i}^{(A)} \leq \mu_{D, i}^{(A)}, \lambda_{U, i}^{(R)} \leq \mu_{U, i}^{(R)}, \lambda_{D, i}^{(R)} \leq \mu_{D, i}^{(R)} \\
& D_{T}(i) \leq D_{\text {req }} \\
& \max \left(d_{i} / 2, d_{i+1} / 2\right) \leq r_{\mathrm{MAX}} \\
& d_{\mathrm{MIN}} \leq d_{i} \leq d_{\mathrm{MAX}}, \text { for } i=1,2, \ldots, n \\
& d_{\mathrm{MIN}} \leq d_{n+1} .
\end{aligned}
$$

\section{Uniform-Spacing Placement Strategy}

According to the uniform-spacing strategy, all the APs have the same separation distance, i.e., $d_{i}=d$. Thus, the optimization problem for this strategy is modified as

$$
\underset{n, d}{\operatorname{MAX}} \frac{(2 n+1) d D_{M}\left(r_{U}+r_{D}\right)}{(2 n+1+\rho)}
$$

subject to

$$
\begin{aligned}
& \lambda_{k} \leq \mu_{k}, \lambda_{D, i}^{(A)} \leq \mu_{D, i}^{(A)}, \lambda_{U, i}^{(R)} \leq \mu_{U, i}^{(R)}, \lambda_{D, i}^{(R)} \leq \mu_{D, i}^{(R)} \\
& D_{T}(i) \leq D_{\text {req }} \\
& d / 2 \leq r_{\text {MAX }} \\
& d_{\text {MIN }} \leq d \leq d_{\text {MAX }} .
\end{aligned}
$$

\section{Throughrut AnALYsis}

This section suggests a throughput model for cluster-based ITS WMN considering bidirectional asymmetric traffic for users operating in the unsaturated situation. The IEEE 802.11a WLAN is used as an example here. 


\section{A. Throughput}

We calculate the throughput (frame service rate) for each node on top of the channel activity concept. From the viewpoint of a particular node, there are five types of channel activities in this wireless network, including the following:

1) successful frame transmission;

2) unsuccessful frame transmission;

3) empty slot, where all nodes are in backoff or idle;

4) successful frame transmission from other nodes;

5) unsuccessful frame transmission from other nodes.

The channel activities can be described by a sequence of activity time slots [19]-[21]. Subject to the backoff procedure, the duration $T_{t}$ for channel activity type $t$ is defined as

$$
\left\{\begin{array}{l}
T_{1}=T_{4}=T_{S} \\
T_{2}=T_{5}=T_{C} \\
T_{3}=\sigma
\end{array}\right.
$$

where $\sigma$ is the duration of an empty slot, and $T_{S}$ and $T_{C}$ are the successful frame transmission time and collision duration, respectively. From the viewpoint of node $k$, the average duration $T_{v, k}$ of activity time slot can be expressed as

$$
T_{v, k}=\sum_{t=1}^{5} \nu_{t, k}, T_{t}
$$

Here, $\nu_{t, k}$ is the probability of channel activity type $t$ observed by node $k$, and clearly, $\sum_{t=1}^{5} \nu_{t, k}=1$. It is noteworthy that $\nu_{1, k}$ also represents the average number of frames successfully delivered by node $k$ in an activity time slot. Therefore, the frame service rate of node $k$ is equal to

$$
\mu_{k}=\frac{\nu_{1, k}}{T_{v, k}} \quad \text { (in frames per second). }
$$

Now, we calculate the channel activity type probability $\nu_{t, k}$. We consider a wireless network with $K$ nodes employing the CSMA MAC protocol to share the channel. Suppose that the frame arrival rate and the service rate of node $k$ are $\lambda_{k}$ and $\mu_{k}$ (in frames per second) for $k=0,1, \ldots, K-1$, respectively. The probability that one node is busy in contending for the channel and sending data is $\rho_{k}=\lambda_{k} / \mu_{k}$ [22], [23]. Then, the channel activity type probability $\nu_{t, k}$ can be calculated as follows.

1) Successful/Unsuccessful Transmission: One node can successfully deliver a frame only if no other node is sending at the same time. Let $\tau_{k}$ be the conditional transmission probability of node $k$ given that node $k$ is busy due to a nonempty queue. The unsuccessful transmission probability $p_{k}$ for the frame from node $k$ is equal to

$$
p_{k}=1-\prod_{j=0, j \neq k}^{K-1}\left(1-\tau_{j} \rho_{j}\right)
$$

where the second term represents the probability that all the other nodes are in backoff or idle due to an empty queue. Hence, given that the considered node is busy, the probability that this node successfully/unsuccessfully sends a frame in an activity slot can be written as

$$
\begin{aligned}
& \nu_{1, k}=\tau_{k}\left(1-p_{k}\right) \\
& \nu_{2, k}=\tau_{k} p_{k} .
\end{aligned}
$$

2) Empty Slot: One node observes an empty slot when all the nodes are silent. From the viewpoint of the considered node, the empty-slot probability can be computed by

$$
\nu_{3, k}=\left(1-\tau_{k}\right) \prod_{j=0, j \neq k}^{K-1}\left(1-\tau_{j} \rho_{j}\right)
$$

where the first term is the probability of the considered node being in backoff, and the second term denotes the probability that all the other nodes are in backoff or idle.

3) Successful/Unsuccessful Transmission From Other Node: Suppose that the considered node is in backoff at the current activity time slot. The probability that at least one node sends a data frame is $p_{\text {otr }, k}=$ $1-\prod_{j=0, j \neq k}^{K-1}\left(1-\tau_{j} \rho_{j}\right)$. Then, the conditional successful transmission probability $p_{\mathrm{os}, k}$ that one frame is successfully delivered from the other node is

$p_{\mathrm{os}, k}=\frac{\sum_{j=0, j \neq k}^{K-1} \tau_{j} \rho_{j} \prod_{j^{\prime}=0, j^{\prime} \neq j, j^{\prime} \neq k}^{K-1}\left(1-\tau_{j^{\prime}} \rho_{j^{\prime}}\right)}{p_{\mathrm{otr}, k}}$

where the numerator is the probability that exactly one frame is transmitted from the other node. Hence, from the viewpoint of the considered node, the probability of an activity time slot containing a successful/unsuccessful frame transmission from the other node can be given as

$$
\begin{aligned}
\nu_{4, k} & =\left(1-\tau_{k}\right) p_{\mathrm{otr}, k} p_{\mathrm{os}, k} \\
\nu_{5, k} & =\left(1-\tau_{k}\right) p_{\mathrm{otr}, k}\left(1-p_{\mathrm{os}, k}\right) .
\end{aligned}
$$

According to the CSMA MAC protocol, the throughput is influenced by the backoff time. Consider a binary exponential backoff procedure with the initial backoff window size of $W$. Let $p_{k}$ be the unsuccessful frame transmission probability of node $k$ as defined in (23), and let $m_{\mathrm{bk}}$ be the maximum backoff stage. The average backoff time of node $k$ is expressed as

$$
\begin{aligned}
\overline{B_{k}}= & \left(1-p_{k}\right) \frac{W-1}{2}+p_{k}\left(1-p_{k}\right) \frac{2 W-1}{2}+\cdots \\
& +p_{k}^{m_{\mathrm{bk}}}\left(1-p_{k}\right) \frac{2^{m_{\mathrm{bk}}} W-1}{2} \\
& +p_{k}^{\left(m_{\mathrm{bk}}+1\right)}\left(1-p_{k}\right) \frac{2^{m_{\mathrm{bk}}} W-1}{2}+\cdots \\
= & \frac{\left[1-p_{k}-p_{k}\left(2 p_{k}\right)^{m_{\mathrm{bk}}}\right] W-\left(1-2 p_{k}\right)}{2\left(1-2 p_{k}\right)} .
\end{aligned}
$$

Since a busy node transmits data frames every $\left(\overline{B_{k}}+1\right)$ slots on average [24], the transmission probability $\tau_{k}$ for the considered node can be written as

$$
\tau_{k}=\frac{1}{\overline{B_{k}}+1}=\frac{2}{1+W+p_{k} W \sum_{i=0}^{m_{\mathrm{bk}}-1}\left(2 p_{k}\right)^{i}} .
$$


From (23) and (31), we can numerically derive the unique solution of $\boldsymbol{\tau}=\left(\tau_{0}, \tau_{1}, \ldots, \tau_{K-1}\right)$ and $\boldsymbol{p}=\left(p_{0}, p_{1}, \ldots\right.$, $\left.p_{K-1}\right)$. Then, from (21)-(31), we can obtain $\boldsymbol{\nu}_{\mathbf{1}}=\left(\nu_{1,0}\right.$, $\left.\nu_{1,1}, \ldots, \nu_{1, K-1}\right)$ and $\boldsymbol{\mu}=\left(\mu_{0}, \mu_{1}, \ldots, \mu_{K-1}\right)$ for the given $\boldsymbol{\lambda}=\left(\lambda_{0}, \lambda_{1}, \ldots, \lambda_{K-1}\right)$.

The hop distance also affects the throughput and the transmission PHY mode. Generally, the radio signal suffers from path loss, shadowing, and multipath fading. Considering these radio channel effects along with a proper fading margin, we assume that the average reception ranges for eight PHY modes in the IEEE 802.11a WLAN are $D_{\kappa}, \kappa=1,2, \ldots, 8$, and $D_{1}>D_{2}>\cdots>D_{8}$. In principle, the node with a shorter separation distance can transmit at a higher data rate. Therefore, the transmission PHY mode $m_{a}$ for the data frame can be determined according to the separation distance $d_{i}$, i.e.,

$$
m_{a}=\kappa, \quad \text { if } D_{\kappa} \geq d_{i}>D_{\kappa+1} \text {. }
$$

Once the transmission PHY modes for data frame $m_{a}$ and control frame $m_{c}$ are given, we can calculate the successful frame transmission time $T_{S}$ and the collision duration $T_{C}$ by

$$
\begin{aligned}
T_{S}= & T_{\mathrm{DATA}}\left(L, m_{a}\right)+\delta+\mathrm{SIFS} \\
& +T_{\mathrm{ACK}}\left(m_{c}\right)+\delta+\mathrm{DIFS} \\
T_{C}= & T_{\mathrm{DATA}}\left(L, m_{a}\right)+\delta+\mathrm{EIFS}
\end{aligned}
$$

where $\delta$ is the propagation delay. The durations of the short interframe space (SIFS), distributed interframe space (DIFS), and extended interframe space $\left(\mathrm{EIFS}=\mathrm{SIFS}+T_{\mathrm{ACK}}\left(m_{c}\right)+\right.$ DIFS) are defined in [25]. $T_{\text {DATA }}\left(L, m_{a}\right)$ is the transmission time of a data frame with payload size $L$ using PHY mode $m_{a}$, and $T_{\mathrm{ACK}}\left(m_{c}\right)$ is that of an acknowledgement (ACK) frame using PHY mode $m_{c} . T_{\mathrm{DATA}}\left(L, m_{a}\right)$ and $T_{\mathrm{ACK}}\left(m_{c}\right)$ can be calculated as in [25].

\section{B. Wireless Access Link Capacity Between AP and User}

In the cell of $A P_{i}$, there are $K=1+l_{i} D_{M}$ nodes (including one AP and $l_{i} D_{M}$ users) contending for the channel. Suppose that all the users have the same uplink frame arrival rate and service rate, i.e., $\lambda_{k}=\lambda_{1}=r_{U} / L$ and $\mu_{k}=\mu_{1}$ for $k=1,2, \ldots, K-1$. In addition, the downlink frame arrival rate and the service rate of $A P_{i}$ are $\lambda_{0}=\lambda_{D, i}^{(A)}=l_{i} D_{M} r_{D} / L$ and $\mu_{0}$, respectively. Thus, the busy probability of user $k$ is $\rho_{k}=\lambda_{1} / \mu_{1}$, and that of the AP is $\rho_{0}=\lambda_{0} / \mu_{0}$. For simplicity, we consider a worst case where all the nodes in a cell transmit at the same data rate. In the cell of $A P_{i}$ with coverage of $l_{i}=\left(d_{i} / 2+d_{i+1} / 2\right)$, the transmission PHY mode $m_{a}$ for the data frame is determined by the maximum possible separation distance between a user and the AP, i.e., $\max \left(d_{i} / 2, d_{i+1} / 2\right)$.

We evaluate the access frame service rates as follows. Let $\tau_{0}$ and $\tau_{1}$ be the transmission probabilities for the AP and a user, respectively. By (23), the unsuccessful transmission probabilities for $\mathrm{AP}\left(p_{0}\right)$ and that for a user $\left(p_{1}\right)$ are equal to

$$
\begin{aligned}
& p_{0}=1-\left(1-\tau_{1} \rho_{1}\right)^{K-1} \\
& p_{1}=1-\left(1-\tau_{0} \rho_{0}\right)\left(1-\tau_{1} \rho_{1}\right)^{K-2} .
\end{aligned}
$$

The channel activity type probability $\nu_{t, k}$ for the AP can be expressed as

$$
\left\{\begin{array}{l}
\nu_{1,0}=\tau_{0}\left(1-p_{0}\right) \\
\nu_{2,0}=\tau_{0} p_{0} \\
\nu_{3,0}=\left(1-\tau_{0}\right)\left(1-\tau_{1} \rho_{1}\right)^{K-1} \\
\nu_{4,0}=\left(1-\tau_{0}\right) p_{\text {otr }, 0} p_{o s, 0} \\
\nu_{5,0}=\left(1-\tau_{0}\right) p_{\text {otr }, 0}\left(1-p_{o s, 0}\right)
\end{array}\right.
$$

and that for a user

$$
\left\{\begin{array}{l}
\nu_{1,1}=\tau_{1}\left(1-p_{1}\right) \\
\nu_{2,1}=\tau_{1} p_{1} \\
\nu_{3,1}=\left(1-\tau_{1}\right)\left(1-\tau_{0} \rho_{0}\right)\left(1-\tau_{1} \rho_{1}\right)^{K-2} \\
\nu_{4,1}=\left(1-\tau_{1}\right) p_{\text {otr }, 1} p_{o s, 1} \\
\nu_{5,1}=\left(1-\tau_{1}\right) p_{\text {otr }, 1}\left(1-p_{o s, 1}\right)
\end{array}\right.
$$

where $p_{\text {otr }, 0}=1-\left(1-\tau_{1} \rho_{1}\right)^{K-1}, \quad$ and $\quad p_{\text {otr }, 1}=1-(1-$ $\left.\tau_{0} \rho_{0}\right)\left(1-\tau_{1} \rho_{1}\right)^{K-2}$. In addition

$$
\begin{aligned}
p_{o s, 0}= & \frac{(K-1) \tau_{1} \rho_{1}\left(1-\tau_{1} \rho_{1}\right)^{K-2}}{p_{\text {otr }, 0}} \\
p_{o s, 1}=\frac{1}{p_{\text {otr }, 1}}[ & \tau_{0} \rho_{0}\left(1-\tau_{1} \rho_{1}\right)^{K-2} \\
& \left.\quad+(K-2) \tau_{1} \rho_{1}\left(1-\tau_{0} \rho_{0}\right)\left(1-\tau_{1} \rho_{1}\right)^{K-3}\right] .
\end{aligned}
$$

Then, according to the developed throughput model in Section IV-A, we can obtain the access frame service rates for the AP and the users, i.e., $\mu_{D, i}^{(A)}=\mu_{0}$ and $\mu_{k}=\mu_{1}$, respectively.

\section{Wireless Relay Link Capacity Between APs}

In the relay link, $A P_{i-1}$ with downlink traffic and $A P_{i}$ with uplink traffic contend for the channel. The transmission PHY mode $m_{a}$ for $A P_{i-1}$ and $A P_{i}$ is determined by the separation distance $d_{i}$ between $A P_{i-1}$ and $A P_{i}$. Let $\lambda_{D}=\lambda_{D, i-1}^{(R)}$ be the downlink relay frame arrival rate of $A P_{i-1}$, and let $\lambda_{U}=\lambda_{U, i}^{(R)}$ be the uplink relay frame arrival rate of $A P_{i}$. Suppose that the relay frame service rates of $A P_{i-1}$ and $A P_{i}$ are $\mu_{D}$ and $\mu_{U}$, respectively. Then, the busy probability of $A P_{i-1}$ is $\rho_{D}=$ $\lambda_{D} / \mu_{D}$, and that of $A P_{i}$ is $\rho_{U}=\lambda_{U} / \mu_{U}$.

Now, we evaluate the relay frame service rates of $A P_{i-1}$ and $A P_{i}$. Let $\tau_{D}$ and $\tau_{U}$ be the transmission probabilities of $A P_{i-1}$ and $A P_{i}$. From (23), the unsuccessful transmission probability of $A P_{i-1}\left(p_{D}\right)$ and that of $A P_{i}\left(p_{U}\right)$ can be expressed as

$$
\begin{aligned}
& p_{D}=1-\left(1-\tau_{U} \rho_{U}\right)=\tau_{U} \rho_{U} \\
& p_{U}=\tau_{D} \rho_{D} .
\end{aligned}
$$

Referring to (24)-(29), the channel activity type probability for $A P_{i-1}$ can be calculated by

$$
\left\{\begin{array}{l}
\nu_{1, D}=\tau_{D}\left(1-p_{D}\right)=\tau_{D}\left(1-\tau_{U} \rho_{U}\right) \\
\nu_{2, D}=\tau_{D} p_{D}=\tau_{D} \tau_{U} \rho_{U} \\
\nu_{3, D}=\left(1-\tau_{D}\right)\left(1-\tau_{U} \rho_{U}\right) \\
\nu_{4, D}=\left(1-\tau_{D}\right) \tau_{U} \rho_{U} \\
\nu_{5, D}=0
\end{array}\right.
$$




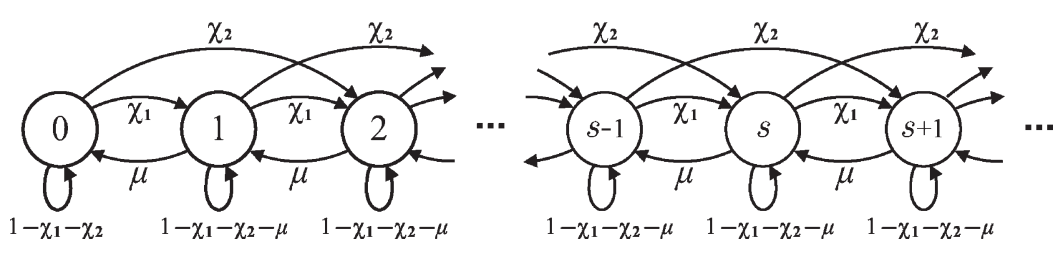

Fig. 5. State transition diagram of the queue for the uplink traffic of an AP, where the state variable $s$ is the number of frames queued in the AP.

and that of $A P_{i}$ are

$$
\left\{\begin{array}{l}
\nu_{1, U}=\tau_{U}\left(1-p_{U}\right)=\tau_{U}\left(1-\tau_{D} \rho_{D}\right) \\
\nu_{2, U}=\tau_{U} p_{U}=\tau_{U} \tau_{D} \rho_{D} \\
\nu_{3, U}=\left(1-\tau_{U}\right)\left(1-\tau_{D} \rho_{D}\right) \\
\nu_{4, U}=\left(1-\tau_{U}\right) \tau_{D} \rho_{D} \\
\nu_{5, U}=0
\end{array}\right.
$$

Then, the relay frame service rate for $A P_{i-1}$ (i.e., $\mu_{D, i-1}^{(R)}=$ $\left.\mu_{D}\right)$ and that for $A P_{i}$ (i.e., $\left.\mu_{U, i}^{(R)}=\mu_{U}\right)$ can be obtained by the developed throughput model in Section IV-A.

\section{Delay Analysis}

This section presents an analytical method to evaluate frame delay and jitter (delay variance) in the considered ITS WMN. The $p$-persistent CSMA MAC protocol is considered, which nicely approximates the standard protocol when the average backoff times are the same [26], [27]. In addition, the parameter $p$ is equal to the transmission probability $\tau_{k}$ for a busy node, as defined in (31). Due to the memoryless backoff time, the $p$-persistent protocol is more tractable.

The considered multihop ITS network can be described by the series queue model [22], where the output frames from a hop node are the input frames to the next hop node. Suppose that the frame arrivals for each end user follow a Poisson process. Following the $p$-persistent CSMA protocol, the frame contention delay (i.e., the frame service time) at each hop is geometrically distributed. Referring to [22, ch. 4.1 and 4.2], the frame interdeparture time at each hop is also geometrically distributed. More importantly, the whole multihop network can be decomposed into individual hops, and each one can be modeled by an independent Markov chain. Hence, we can evaluate the delay and jitter hop by hop.

We consider the queue for the uplink relay traffic of $A P_{i}$ as an example. By (44), we can obtain the probability $\nu_{1}$ that $A P_{i}$ successfully sends one relay frame to $A P_{i-1}$ in an activity slot. In $A P_{i}$, the interface to receive relay frames from $A P_{i+1}$ and the interface to receive access frames from local users independently operate at different channels. Due to the geometric frame service time of $A P_{i+1}$, we assume the geometric interarrival time for the relay frames from $A P_{i+1}$ [22]. We also assume the geometric interarrival time for the access frames from local users. Consider the example in Section IV-B, where one AP and $(K-1)$ users contend for the channel. Following the $p$-persistent CSMA protocol, one busy node transmits its frame independently in each activity slot with probability $\tau_{k}$. The probability that one uplink access frame is successfully delivered to the AP is $p_{S U}=\left(1-\rho_{0} \tau_{0}\right)\left[(K-1) \tau_{1} \rho_{1}(1-\right.$ $\left.\left.\tau_{1} \rho_{1}\right)^{K-2}\right]$, which is also independent in each activity slot.
TABLE II

Notations in DELAY ANALYSiS

\begin{tabular}{ll}
\hline Symbol & Item \\
\hline$s$ & State variable, the number of queued frames \\
$P_{s}$ & Steady-state probability of $s$ frames being queued \\
$p_{s, s^{\prime}}$ & State-transition probability from state $s$ to state $s^{\prime}$ \\
$\chi_{1}, \chi_{2}, \mu$ & State-transition probabilities, \\
& $\left(\chi_{1}, \chi_{2}, \mu\right)=\left(p_{s}, s+1, p_{s}, s+2, p_{s, s-1}\right)$ \\
$\alpha_{R}$ & Arrival probability of relay frame in a slot \\
$\alpha_{L}$ & Arrival probability of local access frame in a slot \\
$h$ & The $h$-th position of the queue of a node \\
$Q_{h}$ & Probability of an incoming frame being placed at the \\
$D_{h}$ & $h$-th position of queue \\
& Time spent for a frame placed at the $h$-th position to \\
$D_{T}(i), \sigma_{T}^{2}(i)$ & be successfully sent \\
$T(k), \sigma^{2}(k)$ & Overall frame delay and variance \\
$T_{D, A}(i), \sigma_{D}^{2}, A$ & Delay and variance for the UL access frame of user $k$ \\
$T_{U, R}(i), \sigma_{U, R}^{2}(i)$ & Delay and variance for the DL access frame of $A P_{i}$ \\
$T_{D, R}(i), \sigma_{D, R}^{2}(i)$ & Delay and variance for the UL relay frame of $A P_{i}$ \\
\hline
\end{tabular}

Thus, it is reasonable to assume the geometric interarrival time for the uplink access frames to $A P_{i}$.

The uplink relay frame and the uplink access frame may concurrently arrive in an activity slot, as shown in Fig. 4. Let $T_{v}$ be the average duration of activity slot for the relay link between $A P_{i-1}$ and $A P_{i}$. Suppose that the relay traffic from $A P_{i+1}$ to $A P_{i}$ is $R_{U, i+1}^{(R)}$ and the aggregated access traffic from local users to $A P_{i}$ is $R_{U, i}^{(A)}$. From the viewpoint of $A P_{i}$, the arrival probability that one uplink relay frame arrives in an activity slot (with average duration $T_{v}$ ) is $\alpha_{R}=\left(R_{U, i+1}^{(R)} / L\right) T_{v}$, where $L$ is the frame payload size. The arrival probability of the uplink access frame in an activity slot is $\alpha_{L}=\left(R_{U, i}^{(A)} / L\right) T_{v}$. Thus, the probability that two frames concurrently arrive in a slot is $\alpha_{L} \alpha_{R}$.

Fig. 5 shows the bulk-arrival queuing model for the uplink relay traffic of $A P_{i}$, where the state variable $s$ is the number of frames queued in the node. The state-transition probabilities are expressed as

$$
\left\{\begin{aligned}
& p_{s, s+2}=\chi_{2}= \alpha_{L} \alpha_{R}\left(1-\nu_{1}\right) \\
& p_{s, s+1}=\chi_{1}= \alpha_{L} \alpha_{R} \nu_{1}+\alpha_{L}\left(1-\alpha_{R}\right)\left(1-\nu_{1}\right) \\
& \quad+\left(1-\alpha_{L}\right) \alpha_{R}\left(1-\nu_{1}\right) \\
& p_{s, s-1}=\mu=\left(1-\alpha_{L}\right)\left(1-\alpha_{R}\right) \nu_{1} \\
& p_{s, s}=1-\chi_{1}-\chi_{2}-\mu
\end{aligned}\right.
$$

where $p_{s, s+2}$ represents the probability that two frames simultaneously arrive, and no queued frame is successfully delivered. We can obtain the steady-state probability $P_{s}$ as detailed in Appendix I. For clarity, Table II summarizes the notations used in the delay analysis.

Now, we evaluate the delay and jitter (delay variance). Consider a frame being placed at the $h$ th position of the firstcome-first-serve (FCFS) queue of a node. The state-transition 


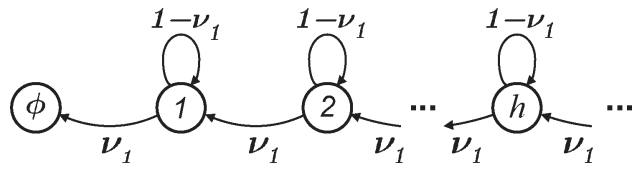

Fig. 6. State transition diagram for the considered frame in a node, where the state variable $h$ denotes the considered frame being placed at the $h$ th position of the queue.

diagram for the considered frame is illustrated in Fig. 6. In the figure, state $h=1$ represents the state that the considered frame is contending for the channel. State $h=\phi$ is defined as the state that the considered frame has been successfully delivered. Let $I_{h}=h^{\prime}$ be the state transition from state $h$ to state $h^{\prime}$ in an activity slot. The state-transition probability can be expressed as

$$
\operatorname{Pr}\left[I_{h}=h^{\prime}\right]= \begin{cases}\nu_{1}, & \text { for } h^{\prime}=(h-1) \\ \left(1-\nu_{1}\right), & \text { for } h^{\prime}=h\end{cases}
$$

First, we deal with the time $D_{h}$ spent for a frame to enter state $\phi$ (i.e., be successfully transmitted), given that this frame is now at state $h$. Clearly

$$
E\left[D_{h} \mid I_{h}\right]= \begin{cases}1+E\left[D_{h-1}\right], & \text { for } I_{h}=(h-1) \\ 1+E\left[D_{h}\right], & \text { for } I_{h}=h\end{cases}
$$

where $D_{h}$ is expressed in activity slots. Therefore, the mean of $D_{h}$ is equal to

$$
\begin{aligned}
E\left[D_{h}\right] & =\sum_{I_{h}} \operatorname{Pr}\left[I_{h}\right] E\left[D_{h} \mid I_{h}\right] \\
& =1+\nu_{1} E\left[D_{h-1}\right]+\left(1-\nu_{1}\right) E\left[D_{h}\right] .
\end{aligned}
$$

Since $E\left[D_{1}\right]=1 / \nu_{1}$, from (48), we can obtain

$$
E\left[D_{h}\right]=\frac{1}{\nu_{1}}+E\left[D_{h-1}\right]=\frac{h}{\nu_{1}} .
$$

By the conditional variance formula [28], the variance of $D_{h}$ can be expressed as

$$
\operatorname{Var}\left(D_{h}\right)=\operatorname{Var}\left(E\left[D_{h} \mid I_{h}\right]\right)+E\left[\operatorname{Var}\left(D_{h} \mid I_{h}\right)\right] .
$$

From (47), it follows that

$$
\begin{aligned}
\operatorname{Var}\left(E\left[D_{h} \mid I_{h}\right]\right) & =\sum_{I_{h}} \operatorname{Pr}\left[I_{h}\right] E\left[D_{h} \mid I_{h}\right]^{2}-\left(E\left[D_{h}\right]\right)^{2} \\
& =\frac{1}{\nu_{1}}-1 .
\end{aligned}
$$

In addition, it is obvious that

$$
E\left[\operatorname{Var}\left(D_{h} \mid I_{h}\right)\right]=\nu_{1} \operatorname{Var}\left(D_{h-1}\right)+\left(1-\nu_{1}\right) \operatorname{Var}\left(D_{h}\right) .
$$

From (50) to (52), we can obtain

$$
\operatorname{Var}\left(D_{h}\right)=\frac{1-\nu_{1}}{\nu_{1}^{2}}+\operatorname{Var}\left(D_{h-1}\right)=\frac{h\left(1-\nu_{1}\right)}{\nu_{1}^{2}}
$$

where the initial condition is $\operatorname{Var}\left(D_{1}\right)=\left(1-\nu_{1}\right) / \nu_{1}^{2}$.
Therefore, the mean and variance of sojourn time for a frame spent in a node can be calculated by

$$
\begin{aligned}
\text { Mean }= & {\left[E\left[D_{h}\right]\right]=\sum_{h=1}^{\infty} Q_{h} E\left[D_{h}\right] } \\
\text { Variance }= & \operatorname{Var}\left(E\left[D_{h}\right]\right)+E\left[\operatorname{Var}\left(D_{h}\right)\right] \\
= & \sum_{h=1}^{\infty} Q_{h}\left(E\left[D_{h}\right]\right)^{2}-\left(E\left[E\left[D_{h}\right]\right]\right)^{2} \\
& +\sum_{h=1}^{\infty} Q_{h} \operatorname{Var}\left(D_{h}\right) .
\end{aligned}
$$

In (54), $Q_{h}$ represents the probability of an incoming frame being placed at the $h$ th position of the FCFS queue at the instant of frame arrival, as detailed in (68) in Appendix II. By (54) and (55), we can obtain the delay $T_{U, R}(i)$ and variance $\sigma_{U, R}^{2}(i)$ for the uplink relay frames of $A P_{i}$, as detailed in Appendix II. By the same method, we also obtain the delay $T(k)$ and variance $\sigma^{2}(k)$ for the uplink access frames of a user, $T_{D, A}(i)$ and $\sigma_{D, A}^{2}(i)$ for the downlink access frames of $A P_{i}$, and $T_{D, R}(i)$ and $\sigma_{D, R}^{2}(i)$ for the downlink relay frames of $A P_{i}$.

This paper considers a two-way overall frame delay between the source user and the central AP. The average overall frame delay $D_{T}(i)$ and variance $\sigma_{T}^{2}(i)$ for a user in the cell of $A P_{i}$ are defined as

$$
\begin{aligned}
D_{T}(i)= & T(k)+T_{D, A}(i) \\
& +\sum_{i^{\prime}=1}^{i}\left(T_{U, R}\left(i^{\prime}\right)+T_{D, R}\left(i^{\prime}-1\right)\right) \\
\sigma_{T}^{2}(i)= & \sigma^{2}(k)+\sigma_{D, A}^{2}(i) \\
& +\sum_{i^{\prime}=1}^{i}\left(\sigma_{U, R}^{2}\left(i^{\prime}\right)+\sigma_{D, R}^{2}\left(i^{\prime}-1\right)\right) .
\end{aligned}
$$

\section{Vi. Performance Evaluations}

In this section, we compare the performances of the increasing-spacing and uniform-spacing strategies. Moreover, we investigate the interactions among delay, capacity, and coverage in ITS WMN. We consider the IEEE 802.11a WLAN with bidirectional asymmetric traffic for each user. The system parameters are summarized in Table III. The demanded uplink and downlink traffic of each user are $r_{U}=0.1 \mathrm{Mb} / \mathrm{s}$ and $r_{D}=$ $0.4 \mathrm{Mb} / \mathrm{s}$, respectively. The ACK control frames are transmitted with PHY mode $m_{c}=1$ for reliability. Assume that all the APs and the users have the same transmission power. Referring to the measured results in [29], we assume that the average reception ranges for eight PHY modes in the IEEE 802.11a WLAN are $D_{\kappa}=\{290,282,267,244,213,167,107,52\}$ (in meters) for the given transmission power. These reception ranges may vary for different environments and transmission power. However, the proposed optimization approach is general enough to evaluate the performances for different wireless ITS networks with various reception ranges.

The maximum reception range in this WMN is assumed to be $\max \left(D_{\kappa}\right)=290 \mathrm{~m}$. Therefore, the maximum separation distance between a user and the AP is constrained by 
TABLE III

SySTEM PARAMETERS FOR PERFORMANCE EVALUATIONS

\begin{tabular}{llc}
\hline & \multicolumn{1}{c}{ Parameters } & Value \\
\hline$D_{M}$ & User density & $0.05 \mathrm{users} / \mathrm{m}$ \\
$r_{U}$ & Demanded uplink traffic per user & $0.1 \mathrm{Mb} / \mathrm{s}$ \\
$r_{D}$ & Demanded downlink traffic per user & $0.4 \mathrm{Mb} / \mathrm{s}$ \\
$r_{M A X}$ & Max. separation distance between user and AP & $290 \mathrm{~m}$ \\
$d_{M I N}$ & Min. separation distance between APs & $200 \mathrm{~m}$ \\
$d_{M A X}$ & Max. separation distance between APs & $290 \mathrm{~m}$ \\
\hline$m_{a}$ & PHY mode for data frame & $654 \mathrm{Mb} / \mathrm{s}$ \\
$m_{C}$ & PHY mode for control frame & $6 \mathrm{Mb} / \mathrm{s}$ \\
$L$ & Data frame payload size & $4067 \mathrm{Bytes}$ \\
$m_{b k}$ & Maximum backoff stage & 6 \\
$W$ & Initial backoff window size & 16 \\
$\sigma$ & Duration of an empty slot & $9 \mu \mathrm{s}$ \\
\hline
\end{tabular}

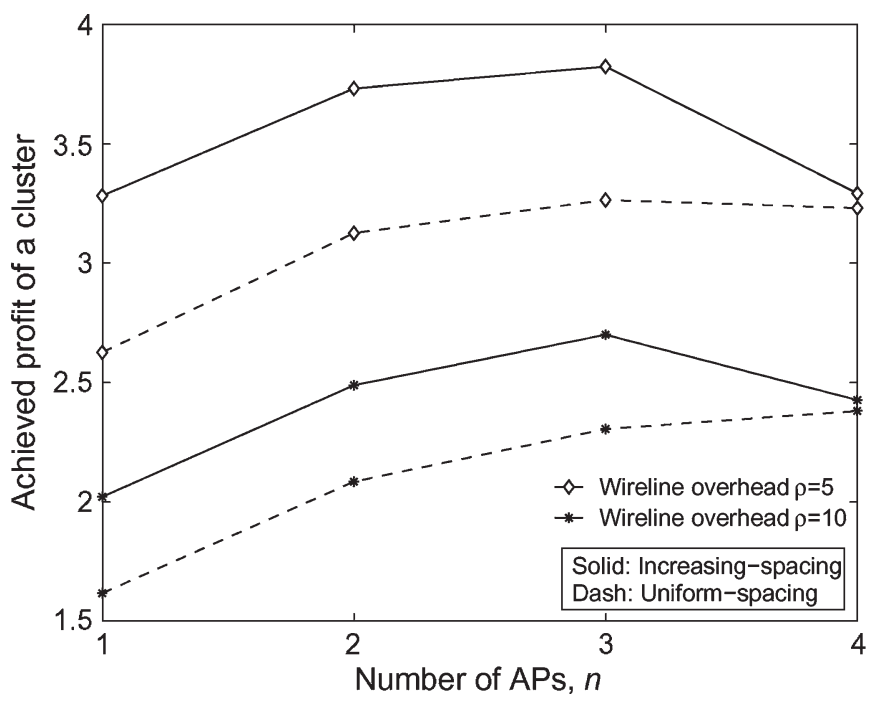

Fig. 7. Achieved profit of a cluster for the increasing-spacing and uniformspacing AP deployment strategies with various wireline overhead $\rho$, where the delay requirement $D_{\text {req }}=0.1 \mathrm{~s}$.

$r_{\mathrm{MAX}}=290 \mathrm{~m}$. In addition, the maximum hop distance of AP is limited to $d_{\mathrm{MAX}}=290 \mathrm{~m}$. This paper also assumes that the minimum reuse distance to avoid cochannel interference is $400(\mathrm{~m})$, which is much larger than the maximum reception range. As shown in Fig. 3, there are two buffer cells between two cochannel cells. To ensure a sufficient reuse distance, the minimum separation distance between APs is limited to $d_{\text {MIN }}=200 \mathrm{~m}$. The minimum reuse distance to avoid cochannel interference will vary with propagation environment, transmission power, and interference tolerance of devices. In other cases, if necessary, we can adopt a greater $d_{\mathrm{MIN}}$ to achieve a larger cochannel reuse distance to avoid interference.

\section{A. Comparison of Increasing-Spacing and Uniform-Spacing Strategies}

Fig. 7 compares the achieved profit of a cluster for the increasing-spacing and uniform-spacing deployment strategies with various wireline overhead $\rho$. The delay requirement is $D_{\text {req }}=0.1$ s. Fig. 7 demonstrates the advantage of the increasing-spacing deployment strategy over the uniformspacing strategy in terms of the achieved profit. In the increasing-spacing strategy, the AP near the central AP uses a shorter hop distance to deliver more traffic load of a cluster, whereas the AP far away from the central AP adopts a longer

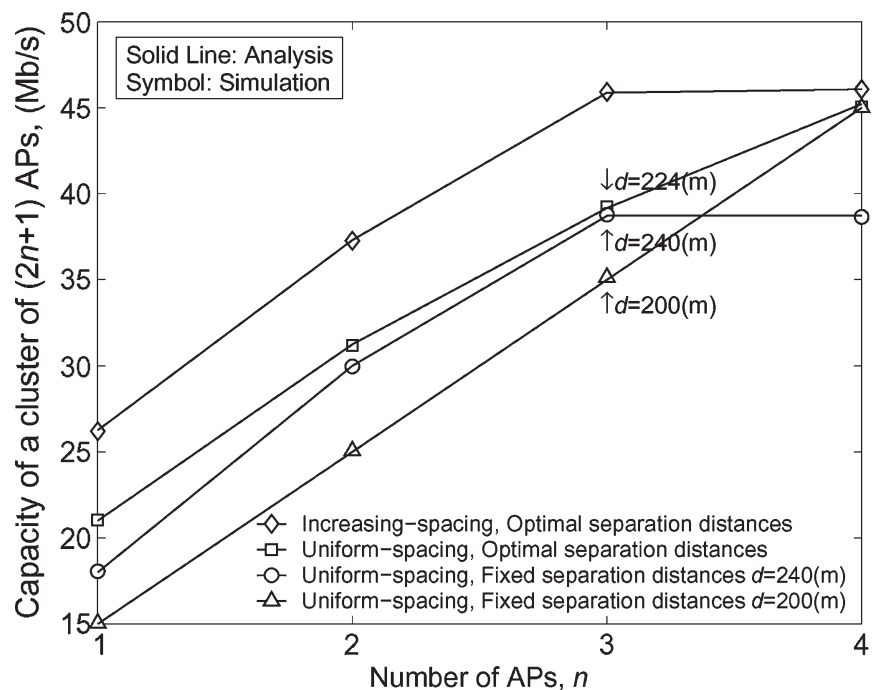

Fig. 8. Capacity of a cluster for the increasing-spacing and uniform-spacing deployment strategies, where the delay requirement $D_{\text {req }}=0.1 \mathrm{~s}$ is set for the cases with optimal separation distances.

hop distance to extend the coverage of a cluster. By the proposed optimization approach to determine the optimal hop distances of APs, the increasing-spacing strategy can achieve better capacity (coverage) performance and higher profit of a cluster.

Fig. 7 also shows that the achieved profit of a cluster is a concave function of the number of APs in a cluster $(n)$. Moreover, there exists an optimal solution of $n$ to maximize the profit of a cluster. For example, with the wireline overhead $\rho=5$, $n=3$ can achieve the optimal profit for both deployment strategies. The corresponding separation distances between APs are $d_{i}=\{200,220,250,496\}$ (in meters) for the increasingspacing strategy and $d=224 \mathrm{~m}$ for the uniform-spacing strategy. In this example, the increasing-spacing strategy can achieve $17 \%$ higher profit of a cluster than the uniformspacing strategy. The optimal number of APs in a cluster may vary for different strategies. For $\rho=10, n=3$ can achieve the optimal profit for the increasing-spacing strategy, whereas $n=4$ for the uniform-spacing strategy. In this case, the achieved profit of a cluster for the increasing-spacing strategy is about $13 \%$ better than that of the uniform-spacing strategy.

Fig. 8 shows the capacity of a cluster for the increasingspacing and uniform-spacing strategies. The optimal separation distances between APs are analytically determined by the optimization approach. The simulation results conform with the analysis results. In Fig. 8, as the number of APs $(n)$ in a cluster increases, the optimal capacity with the increasingspacing strategy increases faster than that with the uniformspacing strategy. However, the increment of capacity gradually diminishes, and even the capacity remains unchanged (see $n=3$ to $n=4$ with the increasing-spacing strategy). Since the profit of a cluster is proportional to the capacity and inversely proportional to the total cost for deploying a cluster as defined in (9), the achieved profit is a concave function of $n$, as shown in Fig. 7.

For comparison, Fig. 8 also shows the achieved capacity for the cases where the separation distances between APs are 


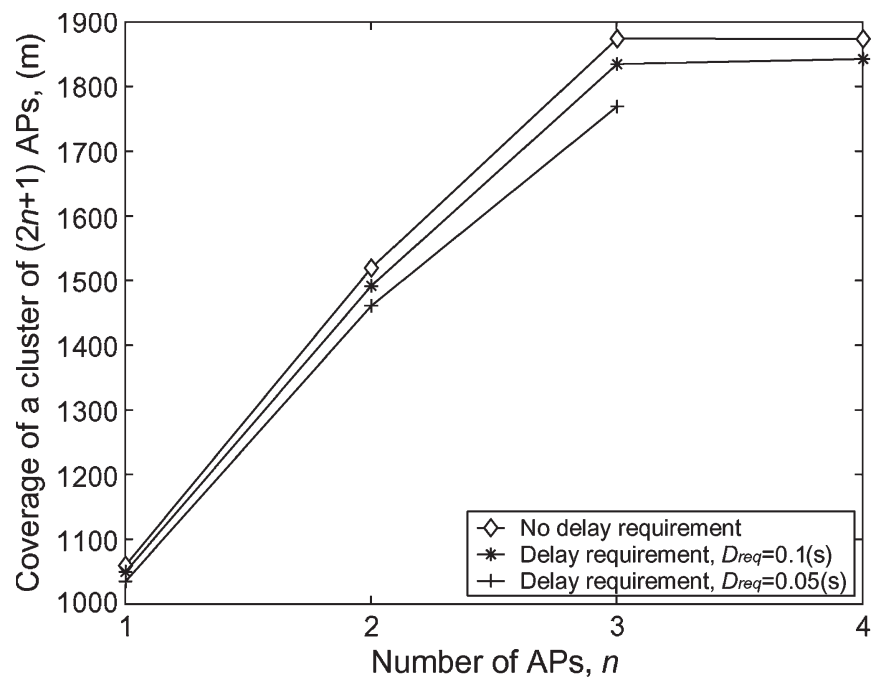

Fig. 9. Coverage of a cluster of APs versus the number of APs $(n)$ in a cluster under different delay requirements, where the increasing-spacing strategy is used.

fixed at $d=200$ and $240 \mathrm{~m}$, respectively. In Fig. 8, since the case with $d=200 \mathrm{~m}$ has a sufficient relay link capacity to accommodate the increasing traffic, the achieved capacity is proportional to the number of APs in a cluster. In addition, due to the larger coverage with more users, the case with $d=240 \mathrm{~m}$ can achieve a higher capacity than the case with $d=200 \mathrm{~m}$ when $n \leq 3$. However, if the number of APs increases from $n=3$ to $n=4$, the achieved capacity for $d=240 \mathrm{~m}$ remains unchanged. This is because the relay link between $A P_{0}$ and $A P_{1}$ is fully utilized. Meanwhile, deploying more APs to extend the coverage with more served users cannot increase the capacity. Noteworthy, although with a smaller coverage, the case with $d=224 \mathrm{~m}$ at $n=3$ has a larger relay link capacity, and thus, it achieves a higher capacity than the case with $d=240 \mathrm{~m}$. This example explains that to achieve the optimal capacity, we should properly design the separation distances between APs to obtain the best tradeoff between the relay link capacity and the coverage.

In these figures, it is demonstrated that the increasingspacing strategy can achieve higher capacity and better profit of a cluster. In addition, the best number of $n$ and the optimal separation distances between APs can analytically be determined by the proposed optimization approach subject to the constraints on delay and throughput.

\section{B. Interactions Among Delay, Coverage, and Capacity}

Figs. 9-11 investigate the interactions among delay, coverage, and capacity, where the increasing-spacing deployment strategy is used. Fig. 9 shows the coverage of a cluster under different delay requirements. In Fig. 9, the optimal coverage at $n=3$ slightly diminishes from 1874 to $1835 \mathrm{~m}$ to meet the delay requirement $D_{\text {req }}=0.1 \mathrm{~s}$. If setting a more stringent delay requirement $D_{\text {req }}=0.05 \mathrm{~s}$, the optimal coverage at $n=$ 3 will further decrease to $1770 \mathrm{~m}$. In this multihop network, slightly reducing the hop distances of APs can improve the relay link capacity. Meanwhile, the smaller coverage of a cluster also lowers the traffic load. Due to higher link capacity and lower

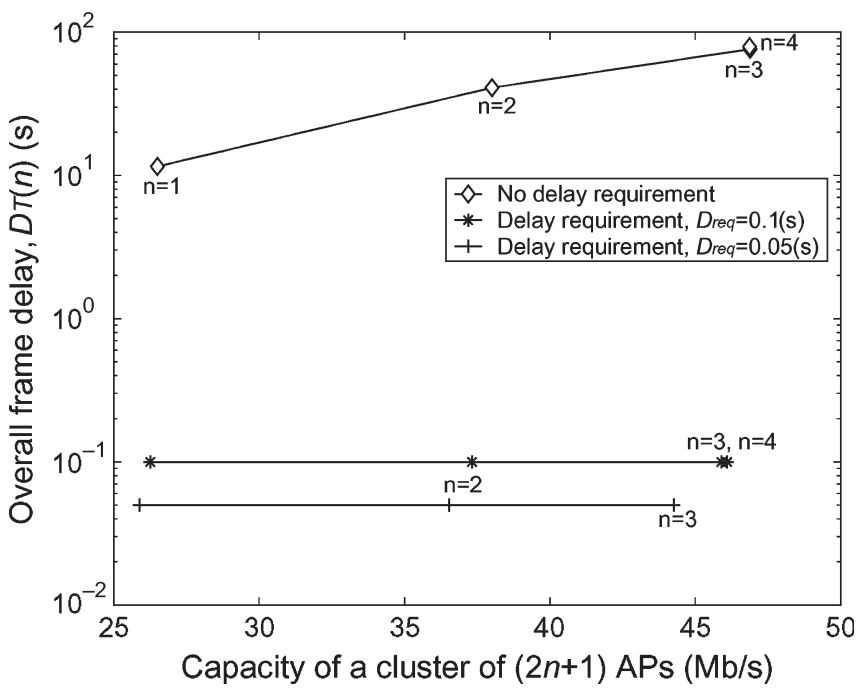

Fig. 10. Overall frame delay versus capacity of a cluster of APs under different delay requirements, where the increasing-spacing strategy is used.

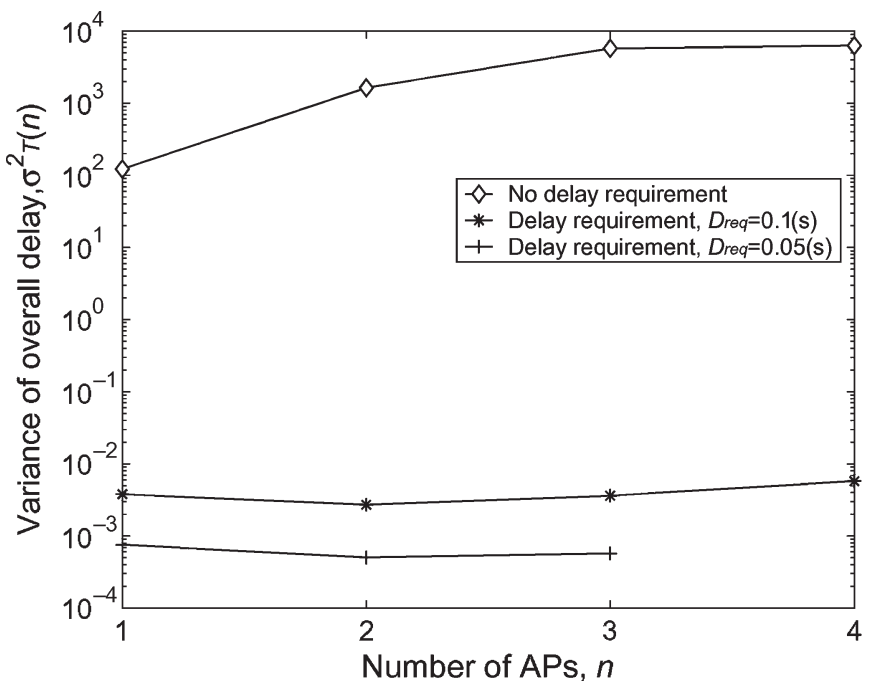

Fig. 11. Overall delay variance (jitter) versus the number of APs $(n)$ in a cluster under different delay requirements, where the increasing-spacing strategy is used.

traffic load, the delay in each link and the overall delay can significantly be improved at the cost of smaller coverage.

Fig. 9 also illustrates that the number of APs $(n)$ in a cluster has a maximum value. Generally, the coverage of a cluster increases as $n$ increases. To accommodate the increasing relay traffic as $n$ increases, we can reduce the hop distances between APs to improve the link capacity. However, the hop distance should be larger than the threshold $d_{\mathrm{MIN}}$ to ensure a sufficient cochannel reuse distance, as defined in (7). Therefore, there exists a maximum value of $n$. In Fig. 9, the maximum number of APs is $n=3$ for the case with $D_{\text {req }}=0.05 \mathrm{~s}$, and $n=4$ for the other cases. In this example, to meet the delay requirement $D_{\text {req }}=0.05 \mathrm{~s}$, the separation distance between $A P_{0}$ and $A P_{1}$ at $n=4$ will be less than $d_{\mathrm{MIN}}$. Since the constraint of (7) cannot be met, no feasible solution exists at $n=4$. In addition, Fig. 9 shows that the coverage remains unchanged for $n=3$ and $n=4$. The coverage of a cluster is actually constrained by the relay link capacity between $A P_{0}$ and $A P_{1}$ since the 
link between $A P_{0}$ and $A P_{1}$ carries more traffic than others. In this example, the separation distance between $A P_{0}$ and $A P_{1}$ for $n=3$ and $n=4$ is equal to the minimum threshold. With the same relay link capacity between $A P_{0}$ and $A P_{1}$, both cases have the same coverage.

Fig. 10 depicts the overall frame delay versus the capacity of a cluster under different delay requirements. At $n=3$, the frame delay can dramatically be improved from 76.4 to $0.1 \mathrm{~s}$, whereas the capacity of a cluster merely decreases from 46.9 to $45.9 \mathrm{Mb} / \mathrm{s}$. The phenomenon of high delay is mainly due to the fact that the relay link between APs is fully utilized if without any delay constraint. Thus, the sojourn time of data frame at the AP will grow toward a large value [22]. However, by properly shortening the hop distances between APs, the relay link capacity and delay can be improved at the expense of a smaller coverage, as shown in Fig. 9. In this example, at $n=3$, the optimal separation distances between APs for the case without any delay requirement are $d_{i}=\{200,229,257,504\}$ (in meters). To guarantee the delay requirement $D_{\text {req }}=0.1 \mathrm{~s}$, the optimal separation distances are reduced to $d_{i}=\{200,220,250,496\}$ (in meters). For both cases, the separation distances between $A P_{0}$ and $A P_{1}$ are equal to $d_{\mathrm{MIN}}=200 \mathrm{~m}$. However, smaller coverage and lower traffic load in the case with $D_{\text {req }}=0.1 \mathrm{~s}$ lead to lower overall frame delay. Fig. 9 also shows that, at $n=3$, the delay requirement $D_{\text {req }}=0.05 \mathrm{~s}$ can be fulfilled at the cost of the optimal capacity decreasing to $44.2 \mathrm{Mb} / \mathrm{s}$.

From Fig. 11, one can observe the variance of overall frame delay against the number of APs in a cluster subject to various delay requirements. In Fig. 11, the case without delay requirement has a relatively high delay variance. However, if the delay requirement $D_{\text {req }}=0.1 \mathrm{~s}$ is set, the delay variance can also be improved to about $5 \times 10^{-3}$. For $D_{\text {req }}=0.05 \mathrm{~s}$, the delay variance can further be controlled to about $6 \times 10^{-4}$.

In Figs. 9-11, we investigate the interactions among delay, capacity, and coverage. It is shown that the optimal capacity and coverage of a cluster can simultaneously be achieved. Moreover, QoS (delay and jitter) can be guaranteed at the expense of lower capacity and coverage.

\section{Model Validation}

The analytical model is validated by simulations. In the simulations, the legacy IEEE 802.11 CSMA protocol with binary exponential backoff scheme is used. We consider a general case where $K^{\prime}$ users and one AP contend for the channel. We also consider the asymmetric traffic for each user. In addition, assume that the time between frame generation in each node is exponentially distributed as in [24].

Fig. 12 illustrates the downlink traffic arrival rate and the achieved service rate in a WLAN with $K^{\prime}$ users, where the transmission rate is $12 \mathrm{Mb} / \mathrm{s}$. Fig. 12 shows the downlink case as an example since the downlink traffic is much higher than the uplink traffic and thereby dominates the network performance. As shown in Fig. 12, the analytical model nicely estimates the service rate if the number of users is larger (e.g., greater than 15 in this example) and slightly underestimates the service rate for the cases with fewer users. This is because the analytical model assumes that the busy probability of each user and the

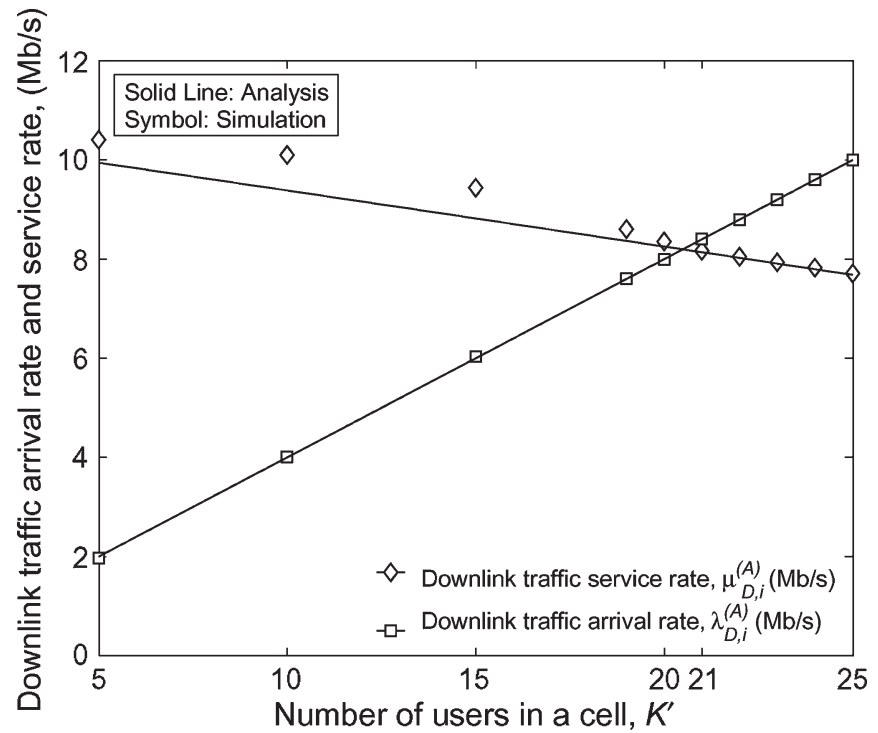

Fig. 12. Downlink traffic arrival rate and achieved service rate in a WLAN with $K^{\prime}$ users and one AP contending for the channel. The transmission rate is $12 \mathrm{Mb} / \mathrm{s}$. For convenience, both the arrival rate and the service rate are expressed in megabits per second.

average number of busy users are fixed all the time. However, in practice, when the AP is idle, the users have more opportunities to successfully deliver their frames and then enter the idle state. Therefore, when the AP becomes busy due to frame arrival, the average number of busy users is actually less than that assumed in the analytical model. With an overestimated collision probability, the analytical model underestimates the service rate. Nevertheless, as the number of users $\left(K^{\prime}\right)$ increases, most of the time, the AP is busy in sending traffic. In this situation, the analytical model can accurately estimate the average number of busy users and the service rate.

Fig. 12 also shows that the analytical model can estimate the accurate upper bound of traffic load in a wireless network with asymmetric traffic. This information is a useful design guideline for network management to guarantee the minimum throughput of each user. In this example, the analysis results suggest that the number of users $\left(K^{\prime}\right)$ should be no more than 20. Obviously, if the number of users increases to $K^{\prime}=21$, the service rate is less than the arrival rate. Meanwhile, the AP operates in the saturated situation, and the number of frames queued in the AP is ever increasing.

Fig. 13 shows the downlink frame delay against the number of users $\left(K^{\prime}\right)$. As shown in Fig. 13, the analytical model well captures the delay behavior if the AP operates near the saturated situation. For example, if the transmission rate is $9 \mathrm{Mb} / \mathrm{s}$, the frame delay will rapidly increase as $K^{\prime} \geq 14$. The analysis results provide a useful insight for network management to avoid intolerable frame delay. According to the analysis results, we can suggest that to meet the frame delay requirement of $0.1 \mathrm{~s}$, the number of users should be no more than 13 if the transmission rate is $9 \mathrm{Mb} / \mathrm{s}$.

In Figs. 12 and 13, it is demonstrated that the analytical model can provide useful information for network management and design. The analytical model nicely estimates the link capacity (i.e., the service rate) in a WLAN with bidirectional 


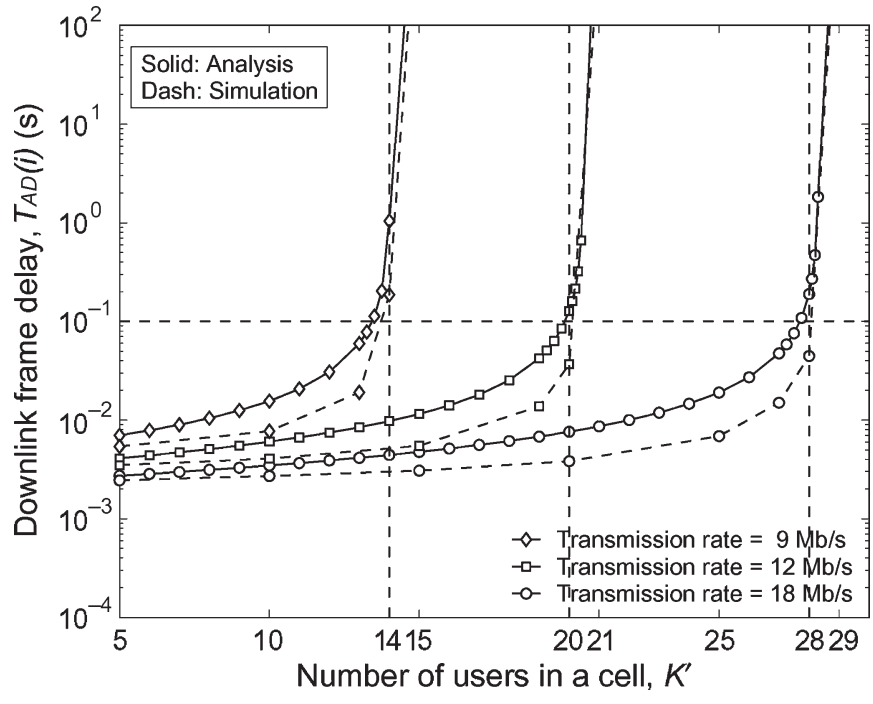

Fig. 13. Downlink frame delay against the number of users $K^{\prime}$ in WLAN, where the transmission rates are 9,12 , and $18 \mathrm{Mb} / \mathrm{s}$, respectively.

asymmetric traffic, although there are some assumptions and approximations. Moreover, the developed model accurately estimates the upper bound of traffic load under the delay requirement. The upper bound of traffic load is an important guideline for network management to determine the system parameters to ensure the QoS of users.

\section{CONCLUSION}

In this paper, we have investigated the AP deployment issue for a scalable ITS WMN considering the overall performances of throughput, coverage, and QoS. The presented mesh network architecture is very suitable for ITS applications due to easy network deployment and lower infrastructure cost. With the QoS requirement, an optimization approach has been proposed to maximize the ratio of total capacity to total cost for deploying a cluster of APs in the considered ITS WMN.

From the system architecture perspective, the proposed WMN has two main advantages. First, the suggested frequency planning can relieve the collision issue and make the WMN more scalable. Second, the proposed network architecture also facilitates the management of QoS and throughput of a WMN. From the system design perspective, this paper has three important elements. First, we have proposed an analytical model to evaluate the throughput, which has considered bidirectional asymmetric traffic for users operating in the unsaturated situation. Second, we have developed a queuing model to analyze the frame delay and jitter. Third, we have applied an optimization approach to determine the best number of APs in a cluster and the optimal separation distances between APs for the proposed ITS WMN. Numerical results have shown that the optimal deployment parameters can analytically be determined, and the goals of throughput enhancement and QoS provisioning can be fulfilled at a slight cost of coverage. In addition, the increasing-spacing strategy outperforms the uniform-spacing strategy in terms of a profit function, considering the capacity, the cost of APs, and the wireline overhead.

\section{APPENDIX I}

Steady-State Probability $P_{s}$, Mean $E[s]$, And VARIANCE $\operatorname{Var}(s)$

The steady-state probability $P_{s}$ is derived by using the probability generating function approach [22]. The generating function $\mathbb{P}(z)$ for the steady-state probability $P_{s}$ is written as

$$
\mathbb{P}(z)=\sum_{s=0}^{\infty} P_{s} z^{s}=\frac{\mu P_{0}}{\mu-\left(\chi_{1}+\chi_{2}\right) z-\chi_{2} z^{2}}
$$

With the condition that $\mathbb{P}(1)=\sum_{s=0}^{\infty} P_{s}=1$, we have

$$
P_{0}=\frac{\mu-\left(\chi_{1}+2 \chi_{2}\right)}{\mu}=1-\frac{\chi_{1}+2 \chi_{2}}{\mu} .
$$

Then, the generating function $\mathbb{P}(z)$ can be rearranged as

$$
\begin{aligned}
\mathbb{P}(z)= & \frac{\mu-\chi_{1}-2 \chi_{2}}{\mu-\left(\chi_{1}+\chi_{2}\right) z-\chi_{2} z^{2}} \\
= & \frac{\mu-\chi_{1}-2 \chi_{2}}{\sqrt{\left(\chi_{1}+\chi_{2}\right)^{2}+4 \mu \chi_{2}}} \\
& \times\left(\frac{1}{z_{1}} \frac{1}{1-z / z_{1}}-\frac{1}{z_{2}} \frac{1}{1-z / z_{2}}\right)
\end{aligned}
$$

where

$$
\begin{aligned}
& z_{1}=\frac{-\left(\chi_{1}+\chi_{2}\right)+\sqrt{\left(\chi_{1}+\chi_{2}\right)^{2}+4 \mu \chi_{2}}}{2 \chi_{2}} \\
& z_{2}=\frac{-\left(\chi_{1}+\chi_{2}\right)-\sqrt{\left(\chi_{1}+\chi_{2}\right)^{2}+4 \mu \chi_{2}}}{2 \chi_{2}} .
\end{aligned}
$$

Since $\sum_{s=0}^{\infty} z^{s}=1 /(1-z)$, it follows that

$$
\begin{aligned}
\mathbb{P}(z)= & \sum_{s=0}^{\infty} P_{s} z^{s} \\
= & \frac{\mu-\chi_{1}-2 \chi_{2}}{\sqrt{\left(\chi_{1}+\chi_{2}\right)^{2}+4 \mu \chi_{2}}} \\
& \times \sum_{s=0}^{\infty}\left[\frac{1}{z_{1}}\left(\frac{z}{z_{1}}\right)^{s}-\frac{1}{z_{2}}\left(\frac{z}{z_{2}}\right)^{s}\right] .
\end{aligned}
$$

By inspecting the coefficient of $z^{s}$, the steady-state probability is obtained as

$$
P_{s}=\frac{\mu-\chi_{1}-2 \chi_{2}}{\sqrt{\left(\chi_{1}+\chi_{2}\right)^{2}+4 \mu \chi_{2}}}\left[\frac{1}{z_{1}^{s+1}}-\frac{1}{z_{2}^{s+1}}\right]
$$

Therefore, the average number $E[s]$ of queued frames in a node can be calculated by

$$
E[s]=\mathbb{P}^{\prime}(1)=\lim _{z \rightarrow 1} \sum_{s=0}^{\infty} s P_{s} z^{s-1}=\frac{\chi_{1}+3 \chi_{2}}{\mu-\chi_{1}-2 \chi_{2}} .
$$


Since $\mathbb{P}^{\prime \prime}(1)=\lim _{z \rightarrow 1} \sum_{s=0}^{\infty} s(s-1) P_{s} z^{s-2}=E\left[s^{2}\right]-E[s]$, the variance $\operatorname{Var}(s)$ is expressed as

$$
\begin{aligned}
\operatorname{Var}(s) & =E\left[s^{2}\right]-(E[s])^{2} \\
& =\mathbb{P}^{\prime \prime}(1)+E[s]-(E[s])^{2} \\
& =\frac{\chi_{1}\left(\mu-\chi_{2}\right)+\left(5 \mu-\chi_{2}\right) \chi_{2}}{\left(u-\chi_{1}-2 \chi_{2}\right)^{2}} .
\end{aligned}
$$

\section{APPENDIX II \\ Average Delay and Variance}

\section{A. Delay and Variance for Uplink Relay Frames of AP}

First, we evaluate the initial probability $Q_{h}$ of an incoming frame being placed at the $h$ th position of the FCFS queue at the arrival instant. In an activity slot, at most two frames will arrive at the queue for the uplink traffic of AP. Let $\beta_{x}$ be the probability that there are $x$ incoming frames at an arbitrary arrival instant. Clearly

$$
\beta_{x}= \begin{cases}\frac{\alpha_{L}\left(1-\alpha_{R}\right)+\alpha_{R}\left(1-\alpha_{L}\right)}{1-\left(1-\alpha_{L}\right)\left(1-\alpha_{R}\right)}=\frac{\alpha_{L}+\alpha_{R}-2 \alpha_{L} \alpha_{R}}{\alpha_{L}+\alpha_{R}-\alpha_{L} \alpha_{R}}, & x=1 \\ 1-\beta_{1}, & x=2 .\end{cases}
$$

At an arbitrary arrival instant, the average number of incoming frames is equal to $\beta_{1}+2 \beta_{2}$. Consider that there are $s$ frames queued at the AP with the probability $P_{s}$. On average, $\left(\beta_{1}+\beta_{2}\right)=1$ incoming frames will be placed at the $(s+1)$ th position of the queue and $\beta_{2}$ incoming frames at the $(s+2)$ th position at an arrival instant. Let $\alpha_{i}$ be the probability of an incoming frame being placed at the $(s+i)$ th position of the queue. Obviously, $\alpha_{1}=\left(\beta_{1}+\beta_{2}\right) /\left(\beta_{1}+2 \beta_{2}\right)$, and $\alpha_{2}=1-$ $\alpha_{1}=\beta_{2} /\left(\beta_{1}+2 \beta_{2}\right)$. Therefore, the initial probability $Q_{h}$ of an incoming frame being placed at the $h$ th position of the queue can be calculated by

$$
Q_{h}= \begin{cases}\alpha_{1} P_{s \mid s=h-1}+\alpha_{2} P_{s \mid s=h-2}, & h \geq 2 \\ \alpha_{1} P_{s \mid s=h-1}, & h=1\end{cases}
$$

Then, we evaluate the average delay $T_{U, R}(i)$ and variance $\sigma_{U, R}^{2}(i)$ for the uplink relay frames of $A P_{i}$. From (54), we obtain

$$
\begin{aligned}
T_{U, R}(i) & =\sum_{h=1}^{\infty} Q_{h} E\left[D_{h}\right] \\
& =\frac{1}{\nu_{1}} \sum_{h=1}^{\infty} h Q_{h} \\
& =\frac{1}{\nu_{1}}\left[\sum_{h=1}^{\infty} h \alpha_{1} P_{s \mid s=h-1}+\sum_{h=2}^{\infty} h \alpha_{2} P_{s \mid s=h-2}\right] \\
& =\frac{1}{\nu_{1}}\left[\alpha_{1} \sum_{s=0}^{\infty}(s+1) P_{s}+\alpha_{2} \sum_{s=0}^{\infty}(s+2) P_{s}\right] \\
& =\frac{1}{\nu_{1}}\left(E[s]+1+\alpha_{2}\right) .
\end{aligned}
$$

Here, $E\left[D_{h}\right]=h / \nu_{1}$ is the average delay for a frame to be successfully transmitted, given that the frame is now at state $h$, as in (49). $P_{s}$ is the probability of $s$ frames being queued at the node. $E[s]=\sum_{s=0}^{\infty} s P_{s}$ is the average number of queued frames at the node, as in (65). According to (55), we have

$$
\begin{aligned}
\sigma_{U, R}^{2}(i) & =\sum_{h=1}^{\infty} Q_{h}\left(E\left[D_{h}\right]\right)^{2}-\left[T_{U, R}(i)\right]^{2}+\sum_{h=1}^{\infty} Q_{h} \operatorname{Var}\left(D_{h}\right) \\
& =\frac{\operatorname{Var}(s)+\left(\alpha_{2}-\alpha_{2}^{2}\right)}{\nu_{1}^{2}}+\frac{\left(1-\nu_{1}\right)\left(E[s]+1+\alpha_{2}\right)}{\nu_{1}^{2}}
\end{aligned}
$$

where $\operatorname{Var}\left(D_{h}\right)=h\left(1-\nu_{1}\right) / \nu_{1}^{2}$ is the variance of $D_{h}$, as in (53), and the variance $\operatorname{Var}(s)$ is detailed in (66).

\section{B. Delay and Variance for Uplink Access Frames of User}

For a user, only one uplink frame will arrive at any arrival instant. Accordingly, $\alpha_{R}=0, \chi_{2}=0$, and $\alpha_{2}=0$. From (64) and (68), the initial probability is equal to $Q_{h}=P_{h-1}=$ $\rho_{c}^{h-1}\left(1-\rho_{c}\right)$, where $\rho_{c}=\chi_{1} / \mu$. By (69) and (70), the average delay $T(k)$ and variance $\sigma^{2}(k)$ for the uplink access frames of a user can be calculated by

$$
\begin{aligned}
T(k) & =\frac{1}{\nu_{1}}(E[s]+1)=\frac{1}{\nu_{1}\left(1-\rho_{c}\right)} \\
\sigma^{2}(k) & =\frac{1}{\nu_{1}^{2}} \operatorname{Var}(s)+\frac{1-\nu_{1}}{\nu_{1}^{2}}(E[s]+1) \\
& =\frac{1-\nu_{1}\left(1-\rho_{c}\right)}{\nu_{1}^{2}\left(1-\rho_{c}\right)^{2}}
\end{aligned}
$$

where, from (65) and (66), $E[s]=\rho_{c} /\left(1-\rho_{c}\right)$, and $\operatorname{Var}(s)=$ $\rho_{c} /\left(1-\rho_{c}\right)^{2}$.

\section{REFERENCES}

[1] R. Pabst et al., "Relay-based deployment concepts for wireless and mobile broadband radio," IEEE Commun. Mag., vol. 42, no. 9, pp. 80-89, Sep. 2004.

[2] I. F. Akyildiz, X. Wang, and W. Wang, "Wireless mesh networks: A survey," Comput. Netw., vol. 47, no. 4, pp. 445-487, Mar. 2005.

[3] J. Jun and M. Sichitiu, "The nominal capacity of wireless mesh networks," IEEE Wireless Commun., vol. 10, no. 5, pp. 8-14, Oct. 2003.

[4] M. Zhang and R. Wolff, "Crossing the digital divide: Cost-effective broadband wireless access for rural and remote areas," IEEE Commun. Mag., vol. 42, no. 2, pp. 99-105, Feb. 2004.

[5] R. Bruno, M. Conti, and E. Gregori, "Mesh networks: Commodity multihop ad hoc networks," IEEE Commun. Mag., vol. 43, no. 2, pp. 123-131, Mar. 2005.

[6] J. Zhu and S. Roy, "MAC for dedicated short range communications in intelligent transportation system," IEEE Commun. Mag., vol. 41, no. 12, pp. 60-67, Dec. 2003.

[7] What is DSRC? [Online]. Available: http:// www.leearmstrong.com/ DSRC/DSRCHomeset.htm

[8] Technical Specification Group Radio Access Networks; RF System Scenarios (Release 1999). 3rd Generation Partnership Project, Rep. 3GPP TR 25.942, V3.0.0, 3GPP.

[9] G. Holland and N. H. Vaidya, "Analysis of TCP performance over mobile ad hoc networks," Wirel. Netw., vol. 8, no. 2/3, pp. 275-288, Mar.-May 2002.

[10] S. Ganguly, V. Navda, K. Kim, A. Kashyap, D. Niculescu, R. Izmailov, S. Hong, and S. R. Das, "Performance optimizations for deploying VoIP services in mesh networks," IEEE J. Sel. Areas Commun., vol. 24, no. 11, pp. 2147-2158, Nov. 2006.

[11] M. Amenetsky and M. Unbehaun, "Coverage planning for outdoor wireless LAN systems," in Proc. IEEE Zurich Semin. Broadband Commun., Feb. 2002, pp. 49.1-49.6.

[12] M. Kobayashi et al., "Optimal access point placement in simultaneous broadcast system using OFDM for indoor wireless LAN," in Proc. IEEE PIMRC, Sep. 2000, pp. 200-204. 
[13] T. Jiang and G. Zhu, "Uniform design simulated annealing for optimal access point placement of high data rate indoor wireless LAN using OFDM," in Proc. IEEE PIMRC, Sep. 2003, pp. 2302-2306.

[14] Y. Lee, K. Kim, and Y. Choi, "Optimization of AP placement and channel assignment in wireless LANs," in Proc. IEEE LCN, Nov. 2002, pp. 831-836.

[15] P. Gupta and P. R. Kumar, "The capacity of wireless networks," IEEE Trans. Inf. Theory, vol. 46, no. 2, pp. 388-404, Mar. 2000.

[16] J. Li et al., "Capacity of ad hoc wireless networks," in Proc. ACM MobiCom, Jul. 2001, pp. 61-69.

[17] J.-H. Huang, L.-C. Wang, and C.-J. Chang, "Deployment strategies of access points for outdoor wireless local area networks," in Proc. IEEE VTC, May 2005, pp. 2949-2953.

[18] MeshDynamics MD4000 Product Family. [Online]. Available: http:// www.meshdynamics.com/prod-md-4000.html

[19] G. Bianchi, "Performance analysis of the IEEE 802.11 distributed coordination function," IEEE J. Sel. Areas Commun., vol. 18, no. 3, pp. 535-547, Mar. 2000.

[20] P. Chatzimisios, A. C. Boucouvalas, and V. Vitsas, "Packet delay analysis of IEEE 802.11 MAC protocol," Electron. Lett., vol. 39, no. 18, pp. 13581359, Sep. 2003.

[21] X. J. Dong and P. Variya, "Saturation throughput analysis of IEEE 802.11 wireless LANs for a lossy channel," IEEE Commun. Lett., vol. 9, no. 2, pp. 100-102, Feb. 2005.

[22] D. Gross and C. M. Harris, Fundamentals of Queueing Theory, 3rd ed. New York: Wiley, 1998.

[23] L. Kleinrock, Queueing Systems: Volume I: Theory. New York: Wiley, 1975.

[24] Y. C. Yay and K. C. Chua, "A capacity analysis for the IEEE 802.11 MAC protocol," Wirel. Netw., vol. 7, no. 2, pp. 159-171, Mar./Apr. 2001.

[25] Part 11: Wireless LAN, Medium Access Control (MAC) and Physical Layer (PHY) Specifications: High-Speed Physical Layer in the $5 \mathrm{GHz}$ Band. supplement to IEEE Std. 802.11, IEEE Std. 802.11a, Sep. 1999.

[26] F. Calì, M. Conti, and E. Gregori, "IEEE 802.11 protocol: Design and performance evaluation of an adaptive backoff mechanism," IEEE J. Sel. Areas Commun., vol. 18, no. 19, pp. 1774-1786, Sep. 2000.

[27] F. Calì, M. Conti, and E. Gregori, "Dynamic tuning of the IEEE 802.11 protocol to achieve a theoretical throughput limit," IEEE/ACM Trans. Netw., vol. 8, no. 6, pp. 785-799, Dec. 2000.

[28] S. M. Ross, Introduction to Probability Models, 5th ed. San Diego, CA: Academic, 1993.

[29] Cisco Aironet 1230AG Series 802.11a/b/g Access Point. [Online]. Available: http://www.cisco.com/

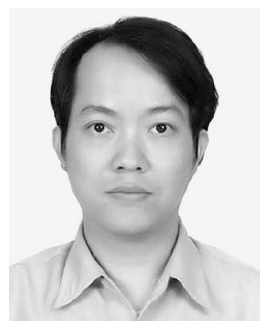

Jane-Hwa Huang received the B.S., M.S., and Ph.D. degrees from the National Cheng Kung University, Tainan, Taiwan, R.O.C., in 1994, 1996, and 2003, respectively, all in electrical engineering.

Since 2004, he has been with the Department of Communication Engineering, National Chiao Tung University, Hsinchu, Taiwan, where he was previously a Postdoctoral Researcher and is currently a Research Assistant Professor. His current research interests are in the areas of performance evaluation, wireless communication networks, wireless multihop communications, and radio resource management.

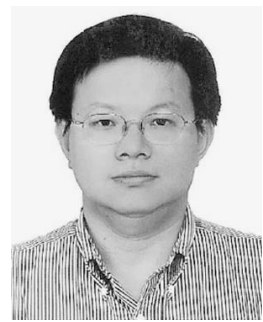

Li-Chun Wang (S'92-M'96-SM'06) received the B.S. degree in electrical engineering from the $\mathrm{Na}-$ tional Chiao Tung University, Hsinchu, Taiwan, R.O.C., in 1986, the M.S. degree in electrical engineering from the National Taiwan University, Taipei, Taiwan, in 1988, and the M.Sc. and Ph.D. degrees in electrical engineering from Georgia Institute of Technology, Atlanta, in 1995 and 1996, respectively.

From 1990 to 1992, he was with the Telecommunications Laboratories, Ministry of Transportation and Communications, Taiwan (currently the Telecom Laboratories, Chunghwa Telecom Company). In 1995, he was with Bell Northern Research of Northern Telecom, Inc., Richardson, TX. From 1996 to 2000, he was with AT\&T Laboratories, where he was a Senior Technical Staff Member with the Wireless Communications Research Department. Since August 2000, he has been an Associate Professor with the Department of Communication Engineering, National Chiao Tung University. He is the holder of a U.S. patent with three more pending. His current research interests are in the areas of cellular architectures, radio-network resource management, cross-layer optimization, and cooperative wireless-communication networks.

Dr. Wang is currently an Associate Editor for the IEEE TRANSACTIONS ON Wireless Communications. He was the corecipient (with G. L. Stuber and C.-T. Lea) of the 1997 IEEE Jack Neubauer Best Paper Award for his paper "Architecture design, frequency planning, and performance analysis for a microcell/macrocell overlaying system," which appeared in the IEEE Transactions on Vehicular Technology (Best Systems Paper published in 1997 by the IEEE Vehicular Technology Society).

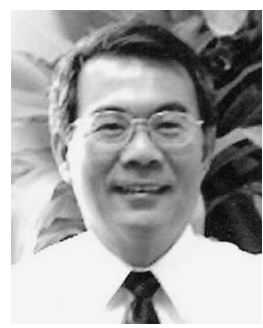

Chung-Ju Chang (S'81-M'85-SM'94-F'05) was born in Taiwan, R.O.C., in August 1950. He received the B.E. and M.E. degrees in electronics engineering from the National Chiao Tung University, Hsinchu, Taiwan, in 1972 and 1976, respectively, and the $\mathrm{Ph} . \mathrm{D}$. degree in electrical engineering from the $\mathrm{Na}-$ tional Taiwan University, Taipei, Taiwan, in 1985.

From 1976 to 1988, he was a Design Engineer, Supervisor, Project Manager, and then Division Director with Telecommunication Laboratories, Directorate General of Telecommunications, Ministry of Communications, Taiwan. He also acted as a Science and Technical Advisor for the Minister of the Ministry of Communications from 1987 to 1989. In 1988, he joined the Faculty of the Department of Communication Engineering, College of Electrical Engineering and Computer Science, National Chiao Tung University, as an Associate Professor, where he has been a Professor since 1993. He was the Director of the Institute of Communication Engineering from August 1993 to July 1995, Chairman of the Department of Communication Engineering from August 1999 to July 2001, and the Dean of the Research and Development Office from August 2002 to July 2004. In addition, he was an Advisor for the Ministry of Education to promote the education of communication science and technologies for colleges and universities in Taiwan during 1995-1999. He is acting as a Committee Member of the Telecommunication Deliberate Body, Taiwan. His research interests include performance evaluation, wireless communication networks, and broadband networks.

Dr. Chang is a member of the Chinese Institute of Engineers (CIE). He serves as an Editor for IEEE COMMUNICATIONS MAGAZINE and as an Associate Editor for the IEEE TRANSACTIONS ON VEHICULAR TECHNOLOGY. 